\title{
SKIN LESION SEGMENTATION TECHNIQUES FOR MELANOMA DIAGNOSIS: COMPARATIVE STUDIES
}

\author{
by \\ Farzad Nowroozipour \\ B.Sc., Sharif University Of Technology, Iran, 2008
}

\begin{abstract}
A project
Presented to Ryerson University

In partial fulfillment of the

Requirements for the degree of

Master of Engineering

In the Program of

Electrical \& Computer Engineering
\end{abstract}

Toronto, Ontario, Canada, 2016

(C) Farzad Nowroozipour 2016 
I hereby declare that I am the sole author of this project. This is a true copy of the project, including any required final revisions, as accepted by my examiners.

I authorize Ryerson University to lend this project to other institutions or individuals for the purpose of scholarly research.

I further authorize Ryerson University to reproduce this project by photocopying or by other means, in total or in part, at the request of other institutions or individuals for the purpose of scholarly research.

I understand that my project may be made electronically available to the public. 


\title{
SKIN LESION SEGMENTATION TECHNIQUES FOR MELANOMA DIAGNOSIS: COMPARATIVE STUDIES
}

\author{
Master of Engineering, 2016 \\ Farzad Nowroozipour \\ Electrical \& Computer Engineering, Ryerson University
}

\begin{abstract}
In recent years, melanoma skin cancer has been one of the rapidest risings of all cancers, which has a high risk of spread. This deadliest form of skin cancer must be diagnosed early for effective treatment. Due to the difficulty and subjectivity of human interpretation, computerized analysis of dermoscopy images has become an important research area. One of the most important steps in dermoscopy image analysis is the segmentation of skin lesion. In this research, we create different new algorithms for the skin lesion segmentation in dermoscopic images. The segmentation algorithms compared are a modified automatic Seeded Region Growing based on Particle swarm optimization image clustering which was used for breast MRI Tumours segmentation, Generalized rough fuzzy c-means algorithm which has been used for brain MR image segmentation, a Support Vector Machine (SVM) and Self-Organizing Map (SOM) with Genetic Algorithm. We used two different datasets with their masks to evaluate the accuracy, sensitivity, and specificity of various segmentation techniques. The results shows that a modified automatic Seeded Region Growing based on Particle swarm optimization image clustering has the highest accuracy (92\%) compares with the other algorithms.
\end{abstract}

Keywords: Computer Aided Diagnosis (CAD); Dermoscopy; Melanoma; Pigment Network; Streaks; Skin Cancer Prevention 


\section{ACKNOWLEDGMENTS}

I wish to thank my supervisor Dr. Kaamran Raahemifar \& Co-supervisor Dr. Vasudevan Lakshminarayanan for supporting and guiding me, sharing their knowledge, helping me learn and grow and become a better researcher over the duration of my Masters program. I will never forget your encouragements and continuous support during my skin cancer research and training. Also my appreciation and my sincere thanks go to my parents, brother and his lovely wife for being all the way with me. I owe them everything.

I would also like to thank Dr. Maryam Sadeghi, the CEO of Meta Optima Technology Inc. \& her team in Vancouver for supporting and cooperation with this project. 
To my wonderful family for their love, support, patience, and encouragement! I'm truly thank you for having them in my life. 


\section{Contents}

$\begin{array}{ll}\text { Declaration } & \text { ii }\end{array}$

Abstract

Acknowledgments

Dedication $\quad$ v

Contents

List of Tables $\quad$ vii

List of Figures $\quad$ ix

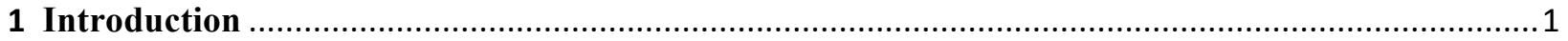

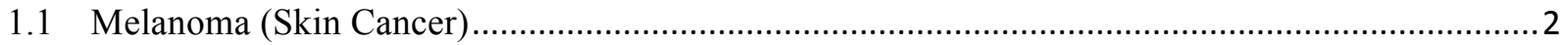

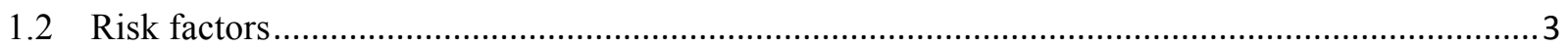

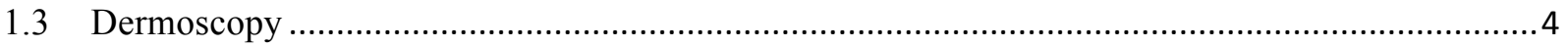

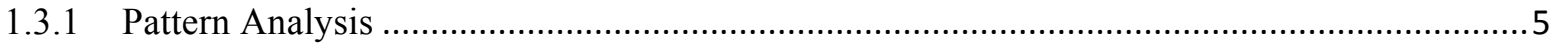

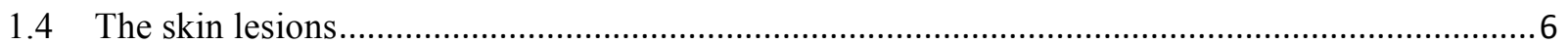

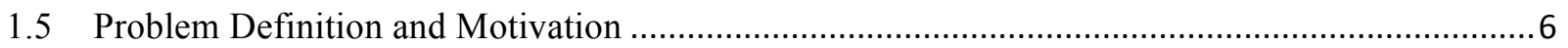

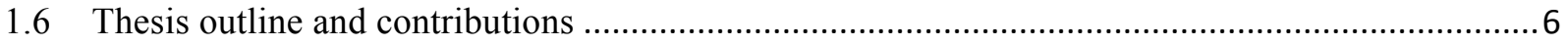

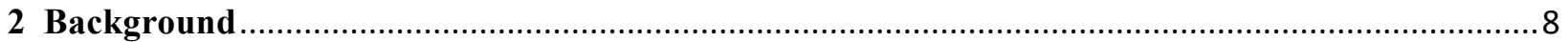

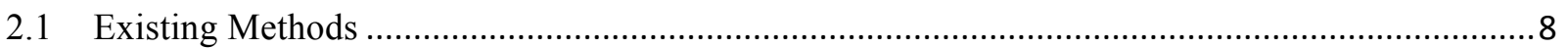

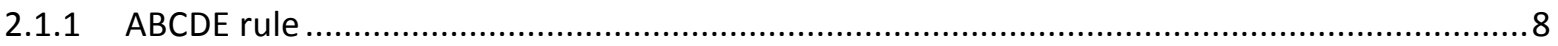

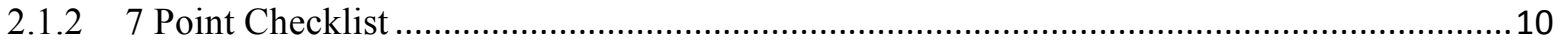

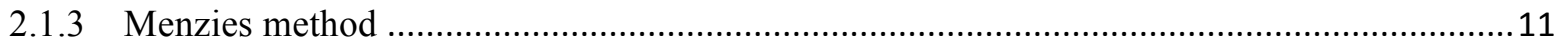

2.1.4 Motivation for automated algorithms …................................................................... 11

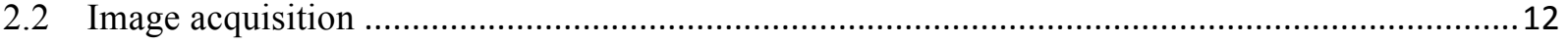

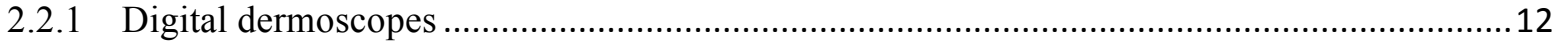

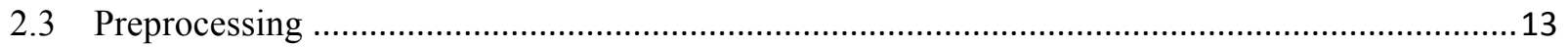

2.3.1 Color Space Transformation …................................................................................. 14

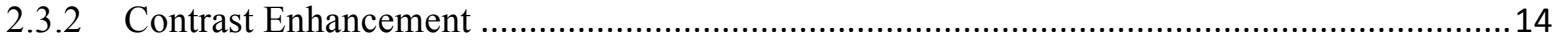

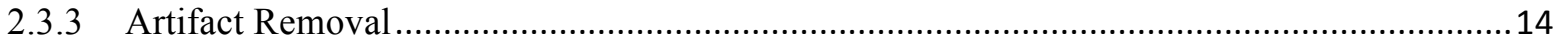




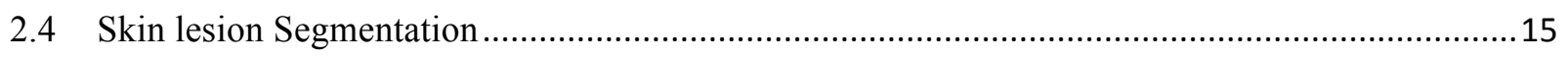

2.4.1 Different types of segmentation methods ....................................................................... 15

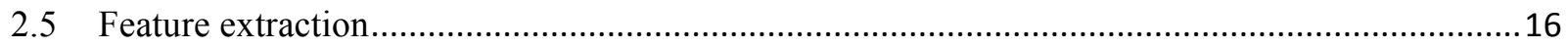

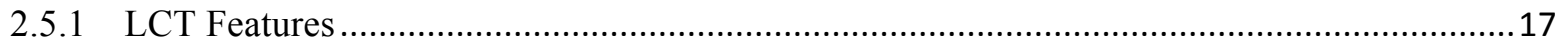

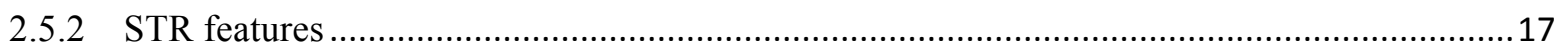

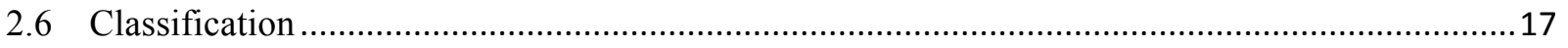

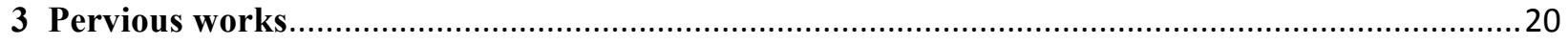

3.1 Melanoma \& non-melanoma skin cancer statistics ....................................................................20

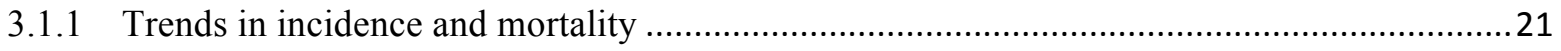

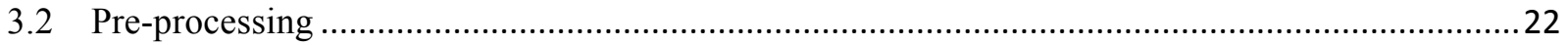

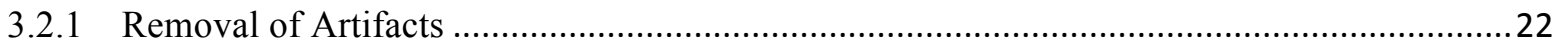

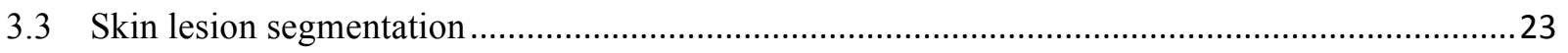

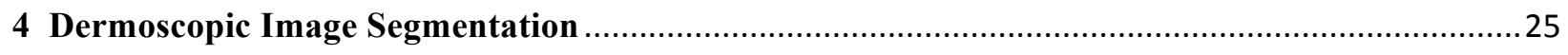

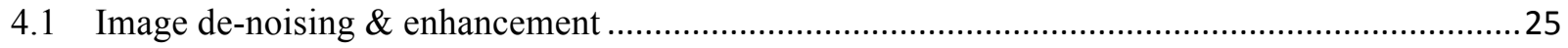

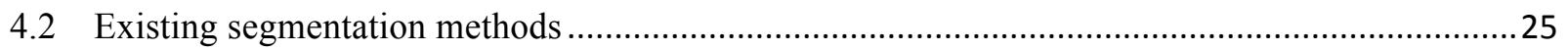

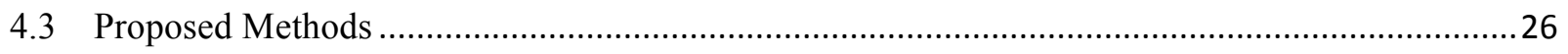

4.3.1 Seeded region growing using particle swarm optimization .................................................27

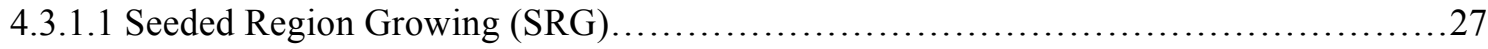

4.3.1.2 Particle Swarm Optimization (PSO) Image Clustering ............................29

4.3.2 Generalized rough fuzzy c-means algorithm …................................................................ 30

4.3.3 Support Vector Machines (SVM) .................................................................................... 31

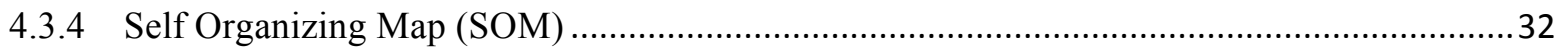

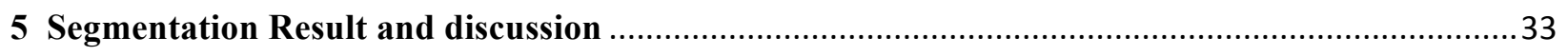

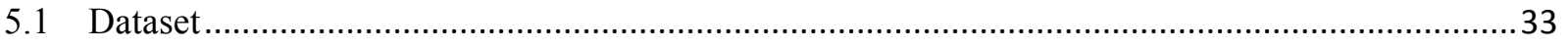

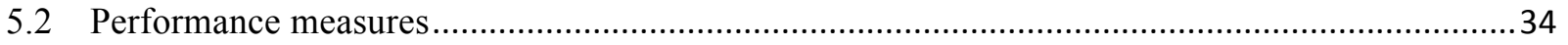

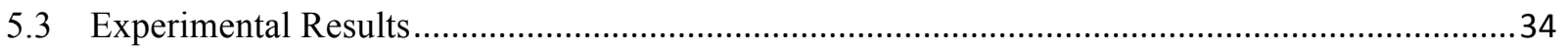

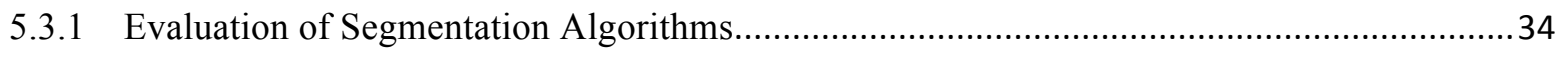

5.3.2 Comparison of the segmentation algorithms …................................................................ 40

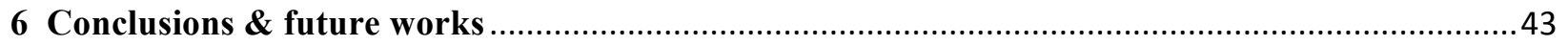

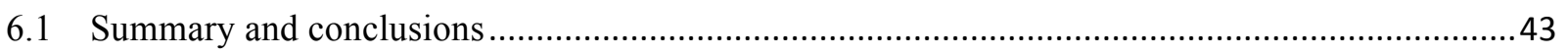

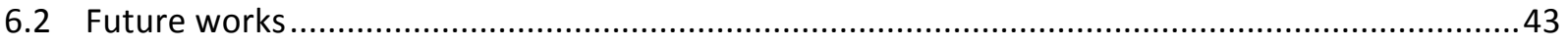

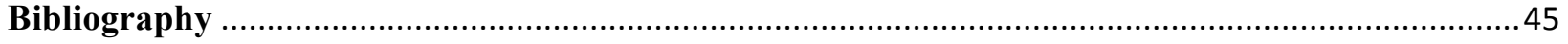




\section{List of Tables}

1.1 Dermoscopic differentiations between benign melanocytic lesions and melanoma using

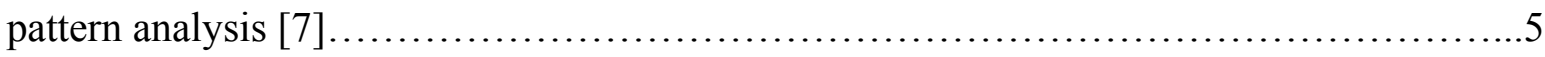

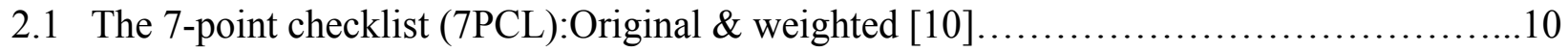

3.1 Melanoma of the skin: Statistics at a glance in Canada [3]..........................20

3.2 Non-melanoma skin cancers (BCC and SCC): Statistics at a glance based on selected

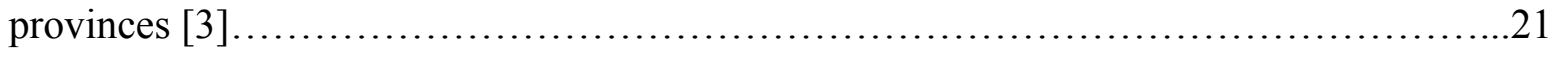

5.1 Comparison table of the segmentation algorithms for Derm database................ 41

5.2 Comparison table of the segmentation algorithms for ELM and IAD database...........42 


\section{List of Figures}

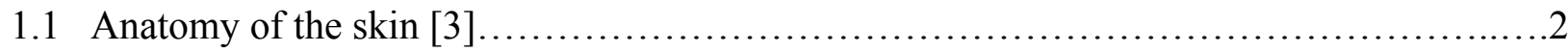

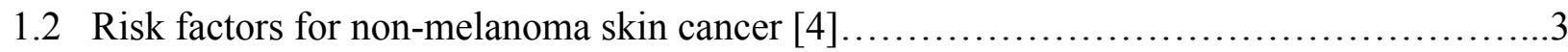

1.3 Dermoscopy images A - The use of a dermatoscope; B,C - Skin lesion [6]...............5

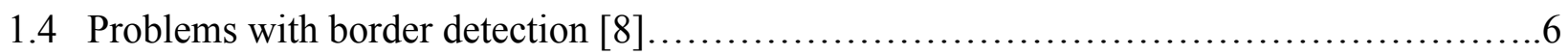

2.1 Asymmetry, Border Irregularity, Color, Diameter \& Evolution (ABCDE rule) [5]..........9

2.2 Block diagram representation for an automated early skin cancer detection system [11]...12

2.3 Figures a, b, c, d, and e show analogue dermoscopes. DinoLite, Handyscope, and DermScope are modern digital dermoscopes shown in $\mathrm{f}, \mathrm{g}$, and $\mathrm{h}$ respectively [7].........13

2.4 Three examples of dermoscopy images with hair (a), oil bubbles (b) and low-contrast

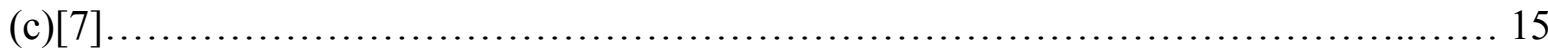

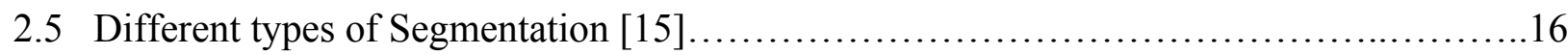

2.6 Examples of (a) Absent, (b) Regular and (c) Irregular streaks [18].....................17

2.7 The dermatoscope device attached to the iPhone and sample of images captured using the

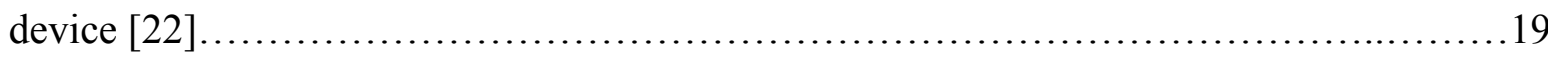

3.1 Age-standardized incidence (1986-2010) and mortality (1986-2009) rates of melanoma of

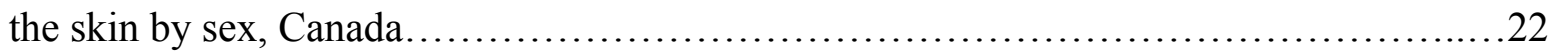

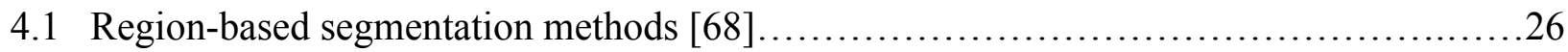

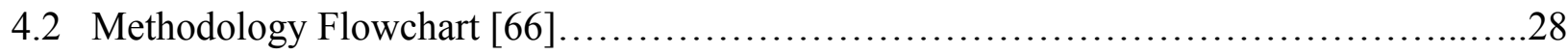

4.3 The Proposed Automatic SRG Initial Seed Selection [66]..........................29

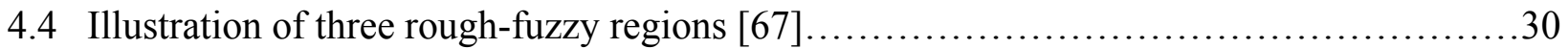


4.5 Two-class SVM is trained on the samples indicated by 'o' and '+' \& One-class SVM is

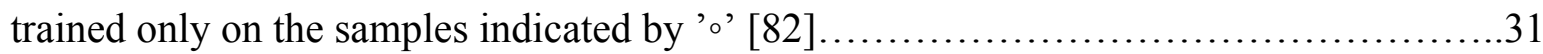

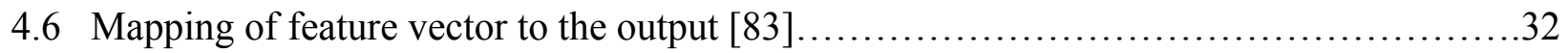

5.1 An epiluminescence microscopy (ELM) image is on the left and its mask is on the right.

5.2 The left one shows the level set evolution of PSO clustering after 189 iteration and the right one shows the initial parameters for the clustering .35

5.3 Original image(a), grayscale image(b), draw the shape to desired position(c), segmentation $\operatorname{result}(\mathrm{d})$ .36

5.4 The procedure of skin lesion segmentation using rough fuzzy c-means algorithm for one ELM image of the database

5.5 The procedure of skin lesion segmentation using rough fuzzy c-means algorithm for one Dermoscopy image of the database.

5.6 The procedure of SOM segmentation is on the left and the training of the SOM network after 200 iteration is on the right.

5.7 The support vector machine procedure for segmenting the skin lesion in two different demoscopy images.

5.8 The Comparison figure of all segmentation algorithms for one dermoscopy image: SRG based on PSO, Rough fuzzy c-means, SOM \& SVM 


\section{Chapter 1}

\section{INTRODUCTION}

We have three type of skin cancer which one of the important one is Melanoma. The occurrence of Malignant melanoma has been increasing during the last couple of years and because there is no impressible treatment for the worst level of melanoma (advanced melanoma), so earlier prediction of skin cancer can be helpful to go through a required treatment $\&$ the lifetime can get more longer [1]. There are some specific symptoms that commonly considered by dermatologists for diagnosing melanoma skin lesion such as: Asymmetry of the lesion shapes, different color variation, irregular of the borders, and specific diameter. The most risk factor of Melanoma is UV radiation and tanning which get so common among the youngsters. The dermatologist can predict the melanoma with $75 \%$ accuracy, which is much lower comparing to Dermoscopy (epiluminescence microscopy) effectiveness. In this regard, a standard digital camera applies using an automated intelligence algorithm to detect the cancerous moles. For further processing, it's too important to detect the exact border of the skin lesion, which is segmentation part. After acquiring the skin lesion images, this part can help to have a better feature extraction of the exact place and also have a high accuracy for classifying the lesion as benign or malignant. There are so many great segmentation algorithms which can have an uncertain outcomes if the preprocessing phase such as illumination, de-noising do not apply on skin lesion images \& Most of them are based on color and texture of the moles. Hence, Using image processing techniques \& creating new algorithms in segmentation part by extracting lesion border from images can help the dermatologists to achieve a high performance and after diagnosing, we can have an efficient treatment to save the human life [2].

This project has categorized as follows: In the next section, I have explained some background of image processing phase of detecting skin cancer $\&$ after, works related to image segmentation are presented. In section 4, I have mentioned the newest and common segmentation algorithms and in section 5, I have discussed about the contribution of this project \& the proposed methods 
are described. Then, I have showed all the experimental results and compared the segmentation algorithms followed by the conclusions and suggestions for future works.

\subsection{MELANOMA (SKIN CANCER)}

Skin cancer is one of the most common \& the deadliest type of cancer among the white skinned population in Europe, North America and Australia. It is the most common cancer is Canada \& in $2014,76,100 \& 6,500$ cases are diagnosed of non-melanoma skin cancer and malignant melanoma respectively. Melanoma is a malignant tumour that starts in melanocytes. Melanocytes are a type of cell that makes melanin, which is the pigment that gives your skin and eyes their color. The meaning of malignant is that it can extend, or to other parts of the body.

There are three main types of skin cancer, regarding to type of the cell. The more common skin cancers are the non-melanoma (NMSC), squamous cell carcinoma (SCC) and basal cell carcinoma (BCC), which are the less deadliest skin cancers comparing to the others. SCC is narrow uniform cells on the surface of the skin, which has shown in Figure 7.1. It also likes to be more offensive than basal cell carcinoma. It is more likely to spread to fatty tissues under the skin, lymph nodes or distant parts of the body [3].

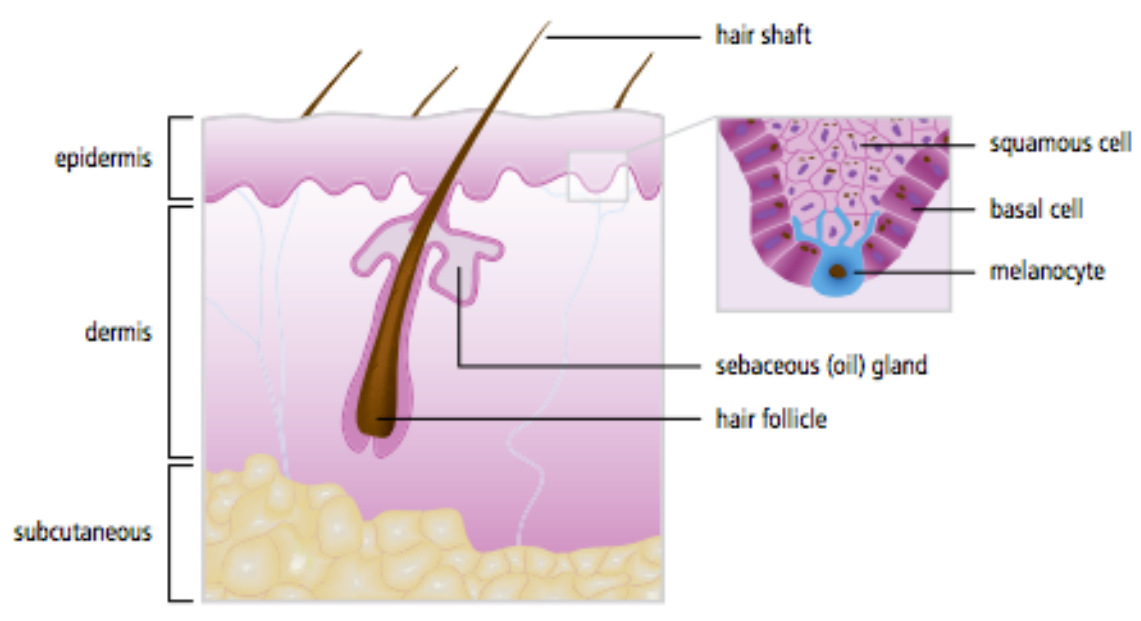

Figure 1.1: Anatomy of the skin [3] 


\subsection{RISK FACTORS}

There are so many factors that can lead to the occurrence of non-melanoma skin cancer (NMSC), which has shown in figure 1.2. A mixture of different factors like Environmental, Genetic, and Phonotypical can affect on the occurrence of Non-melanoma Skin caner that I have discussed about it below [4].

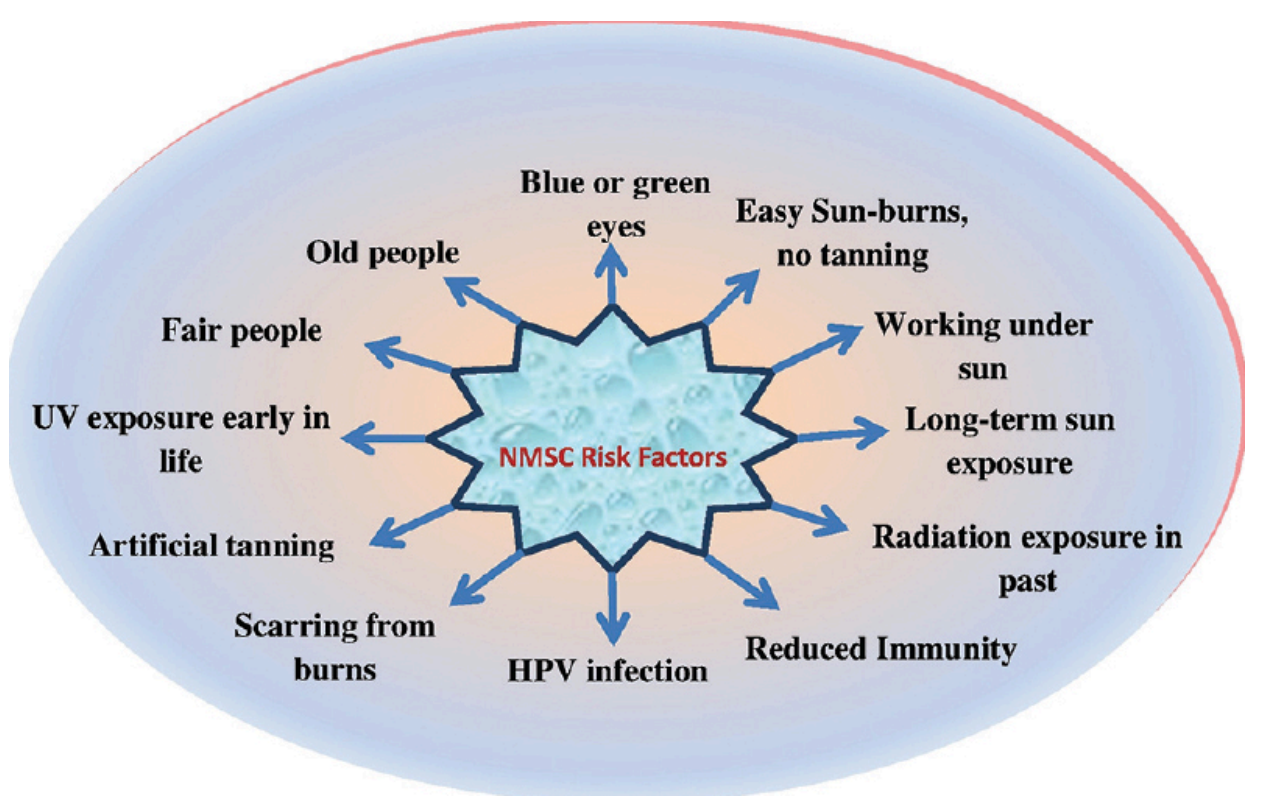

Figure 1.2: Risk factors for non-melanoma skin cancer [4]

\section{Environmental factors:}

Ultraviolet radiation (UVR) is an important risk factor compare to the other. $95 \%$ of the skin cancer is in the transplant patients including SCC \& BCC. SCC is more common that BCC \& it increases during the time. In transplant patients, $75 \%$ of the skin cancer is seeing on head, neck and the other parts of the body which the skin will get damaged by the sun light \& Actinic keratoses (AKs) or solar keratosis will occur in sun-exposed places. Using the chemical elements also can affect the human life. Tanning bed \& sun lamps is similar to UVR but the difference is that it has a higher intensity than the UVR. Most of the research shows that the risk of melanoma in people who are using indoor tanning bed is high. 


\section{Phonotypical factors:}

The amount of pigmentary characteristics is related to the risk of melanoma. The researcher has shown that the fair skin, blue or green eyes, red hair \& Fitzpatrick skin type which burn so more easily are the principal phenotypic risk factor for the occurrence of melanoma.

\section{Genetic factors:}

Having the Family history of melanoma is another risk factor of Melanoma. Genetic mutations are a principal cofactor in the occurrence of NMSC.

Between 5 to 10 percent of Melanoma cased is familial which is more common is the youngsters. A significant proportion of familial melanoma seems to be due to inherited mutations in two tumor suppressor genes: CDKN2A and CDK4 [3]. There are also some disease which increase the risk of melanoma such as: HIV/AIDS lymph proliferative and organ transplantation.

\subsection{DERMOSCOPY}

In the past, most of the physician checked the moles by naked eye and clinical experience but these days dermoscope could help them to assessment \& extract much more information of the lesion which is called dermatoscopy that uses epiluminescence microscopy (ELM) images. It can show you the accurate structure or morphologies for analyzing and give us an accurate result than the past techniques, so now a days most of the dermatologist are using the dermatoscope which is more accurate \& give us the best resolution of the lesion for analyzing which has shown in figure $1.3[6]$.

These days, most of the dermatologist are trying to analysis and recognize the moles from the dermoscopy images like $\mathrm{ABCD}$ rule and 7-point checklist. These algorithms have some advantages and disadvantages that we will talk about it in the next chapter. 


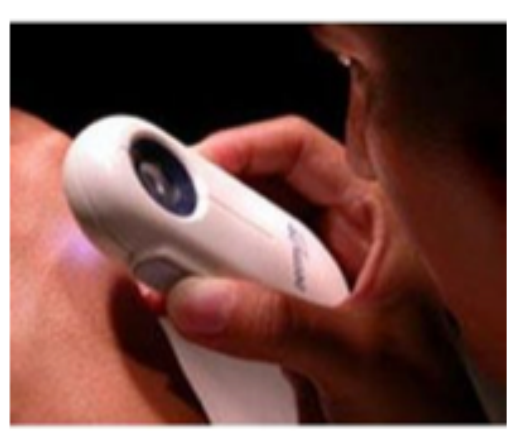

A

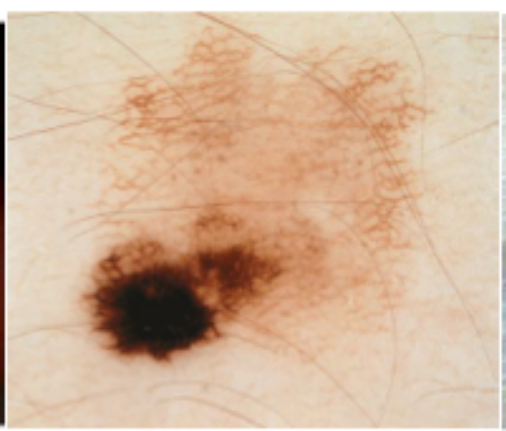

B

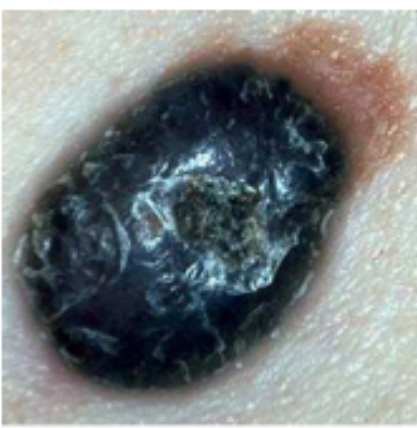

C

Figure 1.3: Dermoscopy images A - The use of a dermatoscope; B,C - Skin lesion [6]

\subsubsection{PATTERN ANALYSIS}

For the first step to analysis the pattern, we need to know that many patterns have dermoscopic structures, which dermatologists are using for recognition of Melanoma, so we use these clinical features about the pattern to have a clinical assessment on the mole. We should first check to see if there is a pigmented structure or not. The features are discriminate by the Colors, Architecture, Symmetric and homogeneity that benign and malignant melanoma has their own characteristics, which has shown in Table 1.1 below [7].

Table 1.1: Dermoscopic differentiations between benign melanocytic lesions and melanoma using pattern analysis [7]

\begin{tabular}{|c|c|c|c|}
\hline $\begin{array}{c}\text { Colours: few vs many } \\
\text { brown, black, red, white, blue } \\
\text { Score 1 point for each colour }\end{array}$ & $\begin{array}{c}\text { Medium } \\
\text { (1-2 points) }\end{array}$ & $\begin{array}{c}\text { H-4 colours } \\
\text { (3-4 points) }\end{array}$ & $\begin{array}{c}\text { 5-6 colours } \\
\text { (5-6 points) }\end{array}$ \\
\hline $\begin{array}{c}\text { Architecture: order vs disorder } \\
\text { Score 0-2 points }\end{array}$ & $\begin{array}{c}\text { None or mild } \\
\text { (no points) }\end{array}$ & $\begin{array}{c}\text { Moderate } \\
\text { (1 point) }\end{array}$ & $\begin{array}{c}\text { Marked } \\
\text { (2 points) }\end{array}$ \\
\hline $\begin{array}{c}\text { Symmetry vs asymmetry } \\
\text { border, colours and structures } \\
\text { Score 0-2 points }\end{array}$ & $\begin{array}{c}\text { Symmetry } \\
\text { in 2 axes } \\
\text { (no points) }\end{array}$ & $\begin{array}{c}\text { Symmetry } \\
\text { in } 1 \text { axis } \\
\text { (1 point) }\end{array}$ & No symmetry \\
Homogeneity vs Heterogeneity \\
$\begin{array}{c}\text { Pigment network, dots/globules } \\
\text { blotches, regression, streaks }\end{array}$ & $\begin{array}{c}\text { one structure } \\
\text { (1 point) }\end{array}$ & $\begin{array}{c}\text { 2 of structure } \\
\text { (2 points) }\end{array}$ & $3 \geq$ structures \\
blue-white veil, polymorphous vessels & & & \\
Score 1 point for each structure & & & \\
\hline
\end{tabular}




\subsection{THE SKIN LESIONS}

One of the most challenges in detection of skin cancer is to create an algorithm to detect the border of the skin lesion automatically cause each lesion has its own structure and some of the challenges which has shown in figure 1.4 are the existence of hair, oil, bubble in the images, irregular borders, low contrast, polychromatic color of the lesion \& fragmentation, so in this article we present different algorithms to detect the lesion border automatically and so fast to have an accurate feature extraction for the next steps [8].

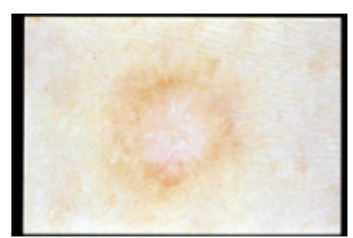

(a) Low contrast

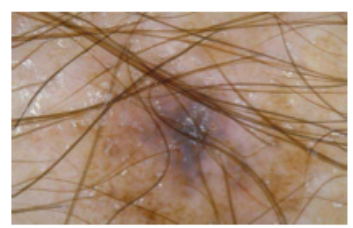

(e) Hairs

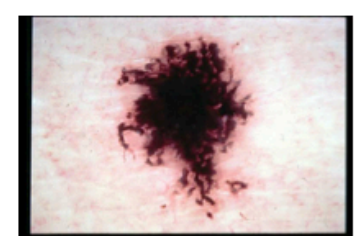

(b) Irregular border

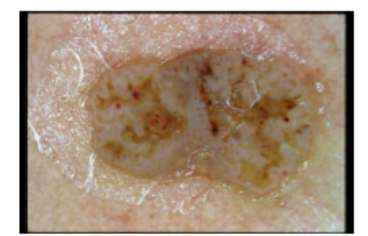

(f) Bubbles

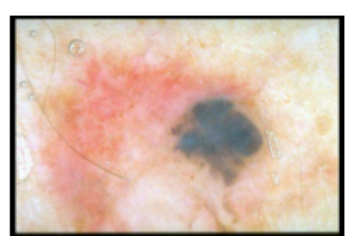

(c) Fuzzy border

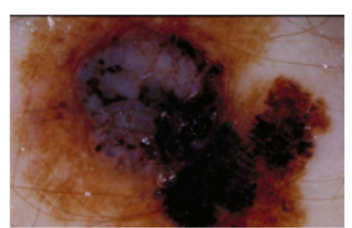

(g) Variegated coloring

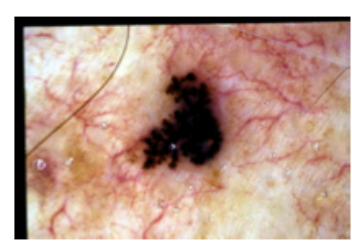

(d) Blood vessels

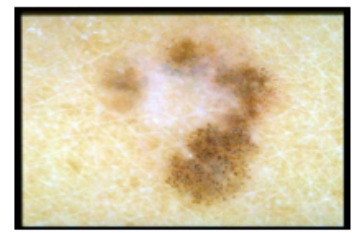

(h) Fragmentation

Figure 1.4: Problems with border detection [8]

\subsection{PROBLEM DEFINITION AND MOTIVATION}

Regarding to all we mentioned in this chapter, the major step in detection of melanoma is the segmentation part, which can affect the other steps like feature extraction, selection and classification to get the result, so creating and implementing an automated segmentation algorithm o segment the lesion from the skin is an important motivation in this project.

\subsection{THESIS OUTLINE AND CONTRIBUTIONS}

The goal in this project is to research and create different automated segmentation algorithms, which have been used in the other disease like breast MRI tumours or brain MR images and 
combined different new segmentation algorithms to have a better performance in segmentation section.

The purpose of this thesis is to propose a novel skin lesion segmentation algorithm, which is part of an automated melanoma screening system. In this thesis, there are three main contributions:

$>$ First having a preprocessing phase on dermoscopy images such as using low pass filter to reduction the noise or Laplacian of Gaussian (LOG) or high pass filter for sharpening the images or remove some artifacts like oil, hair \& bubble to have a better Images.

Creating and Implementing different segmentation algorithm which has been used for the other disease Such as Brain MR Image Segmentation Using Self Organizing Map, Generalized rough fuzzy c-means algorithm for brain MR image segmentation or Breast MRI Tumours Segmentation using Modified Automatic Seeded Region Growing Based on Particle Swarm Optimization Image Clustering.

$>$ A Hybrid image segmentation technique such as seed region growing base on PSO algorithm 


\section{Chapter 2}

\section{BACKGROUND}

\subsection{EXISTING METHODS}

These days, the dermatologist uses different algorithm for analyzing and assessment the Skin lesion. In pervious chapter, I have pointed to the oldest one that was pattern analysis $\&$ also we talked about the specific characteristics of benign and malignant melanoma that helping us to categorized the different types of melanoma. In this part, I have discussed about the other algorithms that most of the physicians and dermatologist use for analyzing the skin lesion such as: ABCDE rule, 7-point checklist and Menzies method.

\subsubsection{ABCDE RULE}

The ABCDE feature extraction is a common method, which can help the dermatologist to assess the skin lesion to check the risk of having malignant melanoma, or not! ABCDE is checking for parameters in the mole such as: Asymmetry, border, color and diameter \& Evolution [5,9].

1) Asymmetry: This indicator is too important to understand about the type of the lesion. For measuring the asymmetry score of the lesion we should separate the image in to major axis and minor axis

2) Border irregularity: The irregularity of the mole can give us the idea of having cancer. To calculate the border, it is divided to eight sections and the sharp pattern will get the score of 1 , the maximum and the minimum irregular border will get 8 and 0 respectively.

3) Colors: for diagnosing of melanoma, we should focus on six different colors include white, red, light brown, dark brown, dark blue \& black, so we should get the score 1 when we see each of these colors. The score is 6 , if we have all the colors and the minimum is 0 . 


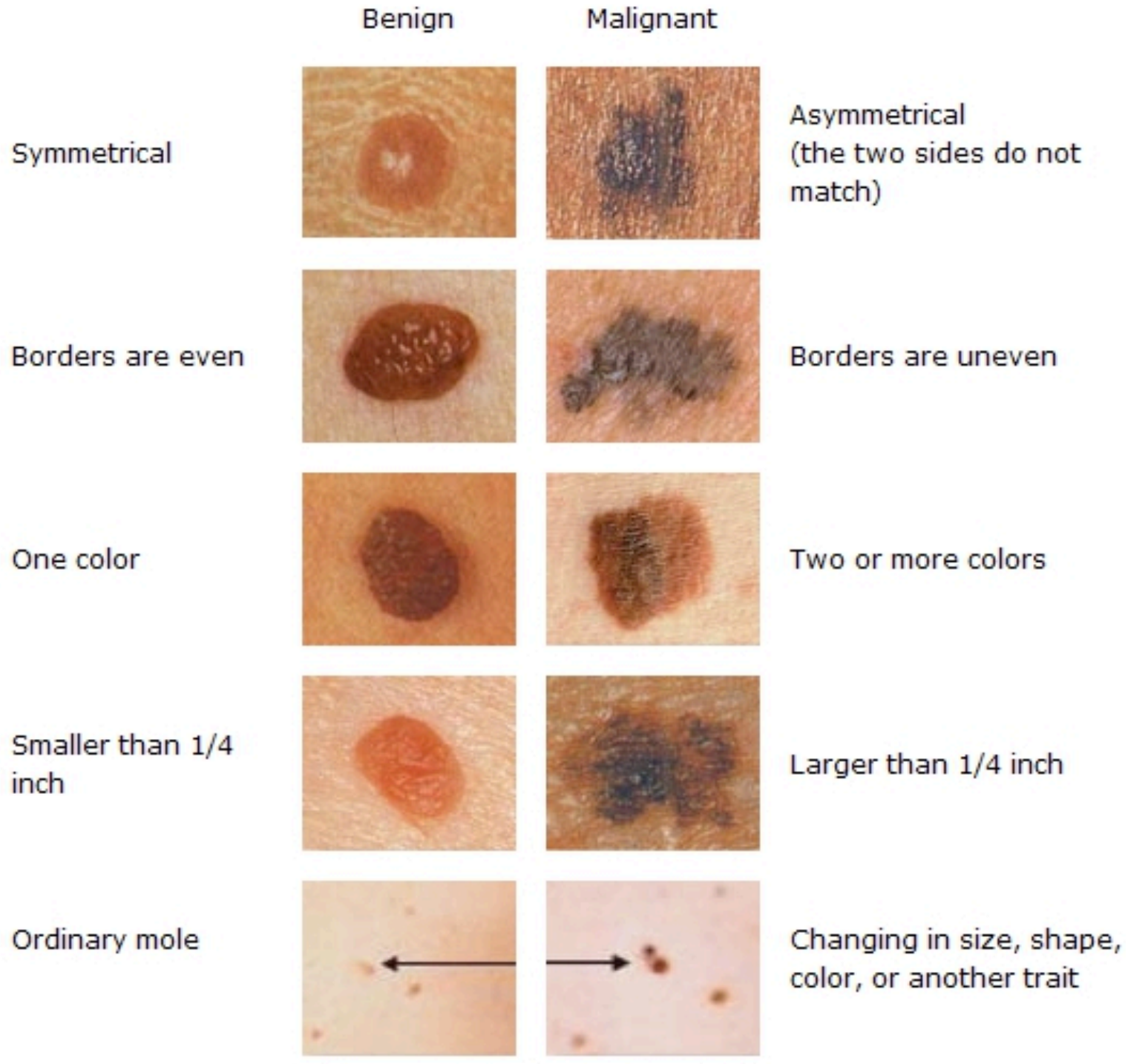

Figure 2.1: Asymmetry, Border Irregularity, Color, Diameter \& Evolution (ABCDE rule) [5]

4) Diameter: depending on the scales, we can find the exact diameter of the skin lesion \& the diameter that is greater than 6 are more likely to be malignant melanoma than the small one. At the end, the TDS can give us a score base on all four parameters, which based on that we make a decision. If the score is less than 4.75 ,then we can say it's a benign skin lesion, for TDS between 4.75 and 5.45,we are suspicious to melanoma and if the TDS is greater than 5.45, we are highly suspicious to melanoma.

$\mathrm{TDS}=1.3 * \mathrm{~A}+0.1 * \mathrm{~B}+0.5 * \mathrm{C}+0.5 * \mathrm{D}$ 
5) Evolution: check the evolution and changing of the mole in size, shape and color during the time.

\subsubsection{POINT CHECKLIST}

In this methods, we have 7 features that each of them will get score 1 and if a skin lesion have a score more than 3,then the lesion is more likely a melanoma and we should go to see a dermatologist. These 7 point check list is determined based on changing in color, shape, size which are the important sign and four minor sign include: inflammation, sensory change, diameter \& crusting. The weighted 7-point checklist was suggested to use by all the specialist and professionals for analyzing the skin lesion. The table 2.1 shows the original and the weighted 7-point checklist [10].

Table 2.1: The 7 point checklist (7PCL):Original \& weighted [10]

\begin{tabular}{ll}
\hline $\begin{array}{l}\text { Original 7PCL } \\
\text { (Score of } \geq \mathbf{3} \text { suggests referral) }\end{array}$ & $\begin{array}{l}\text { Weighted 7PCL } \\
\text { (Score of } \geq \mathbf{3} \text { suggests referral) }\end{array}$ \\
\hline All features (equal weighting) & Major features (2 points) \\
- Change in size of lesion & - Change in size of lesion \\
- Irregular pigmentation & - Irregular pigmentation \\
- Irregular border & - Irregular border \\
- Inflammation & \\
- Itch or altered sensation & Minor features (1 point) \\
- Larger than other lesions (diameter & - Inflammation \\
$>7 \mathrm{~mm}$ ) & - Itch or altered sensation \\
- Oozing/crusting of lesion & - Larger than other lesions (diameter \\
& $>7 \mathrm{~mm})$ \\
& - Oozing/crusting of lesion \\
\hline
\end{tabular}




\subsubsection{MENZIES METHOD}

This method is another way to analysis the skin lesion by two feature set of negative and positive one. A negative set include, having a single color and the positive is consist of 9 feature set, so if we don't have any of these feature sets like a negative sign or at least one of the positive sign, then we will be diagnosed with melanoma.

\subsubsection{MOTIVATION FOR AUTOMATED ALGORITHMS}

All the methods that mentioned in the above are a good way for diagnosing the melanoma but they also have some disadvantaged that should be considered such as:

First of all, it takes a lot of time that all these algorithms and parameters to be exploited for each lesion, so it's not a good way to use them for a large scale of different lesions. Secondly, we can't access to dermatologists and specialists in all over the world like rural areas and in these places, please just go to check by a physician that doesn't have more knowledge about dermatology. More over, there is different opinion and idea about assessment of the parameters and having different methods for recognizing the skin lesion. According to all these problems, we need to research and create automated new algorithms that is more flexible, fast and intelligence to diagnosing the melanoma. To create an Automated algorithm for analyzing the dermoscopy images of the skin lesion we have different stage. I have shown the block diagram for an automated skin cancer detection system in Figure 2.2 that I will discuss about each block in this chapter. 


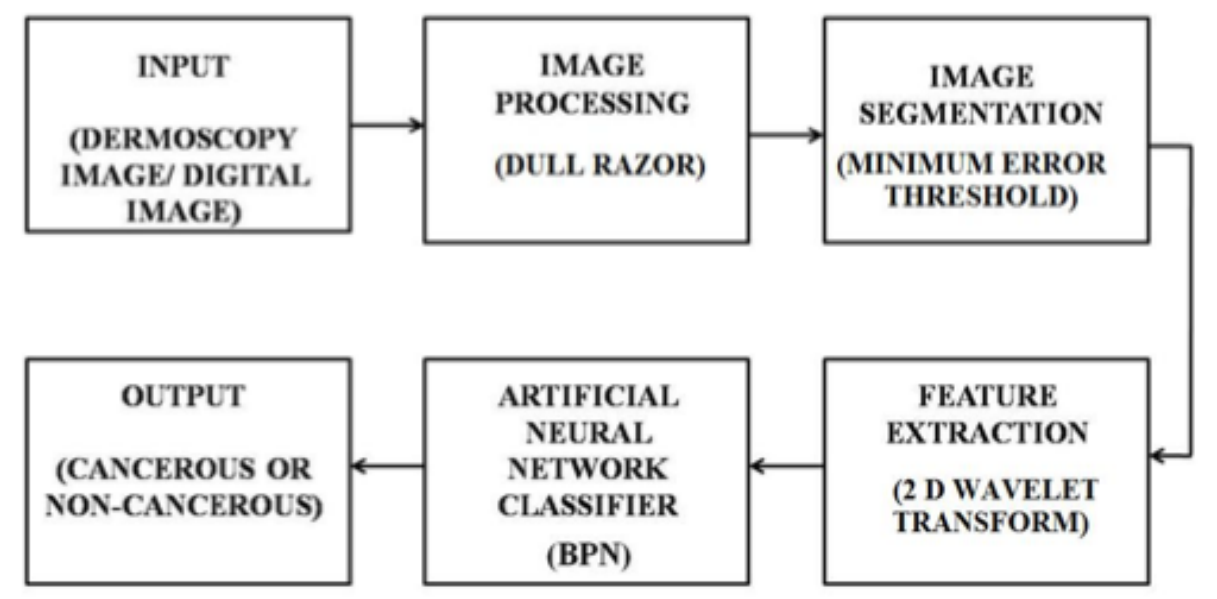

Figure 2.2: Block diagram representation for an automated early skin cancer detection system [11]

\subsection{IMAGE ACQUISITION}

We have different kind of methods in image acquisition for screening the skin lesion such as: Photography, Dermoscopy, Multispectral imaging, Laser-based enhanced diagnosis, Optical coherence tomography, Ultrasound imaging \& magnetic resonance imaging that each of them has their own advantages and limitations [12].

\subsubsection{DIGITAL DERMOSCOPES}

We have different kind of dermoscopes that can give us a high resolution of the skin lesion to use it for the other stages. In this regard, we have both digital and analogue dermoscopes. Working with digital is easier to save all the information and use it for the next steps. Figure 2.3 from a to e, we have shown the analogue dermoscopes that can attached to the camera for taking a photo with high resolution except a. More over, $\mathrm{F}$ and $\mathrm{h}$ also shows the digital dermoscopes as well. D, $\mathrm{f}, \mathrm{g} \& \mathrm{~h}$ show the advanced digital dermoscopes that most of them can attached to the mobile and all the images can send to the specialist with high resolution [7]. 


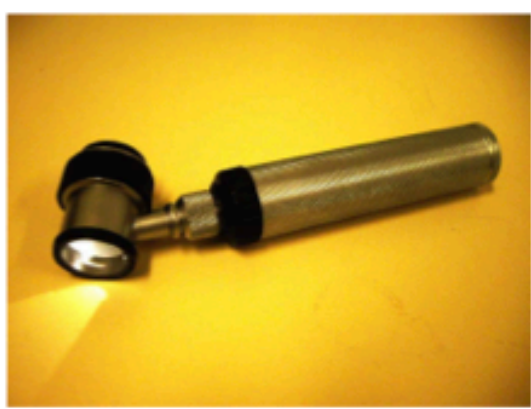

(a) Heine10@

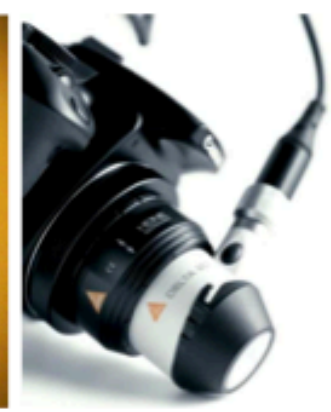

(b) Heine20@

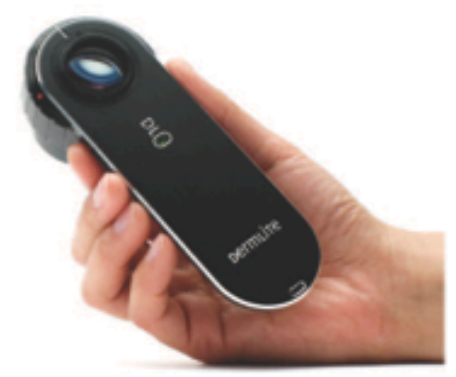

(c) DermLite III DL3®

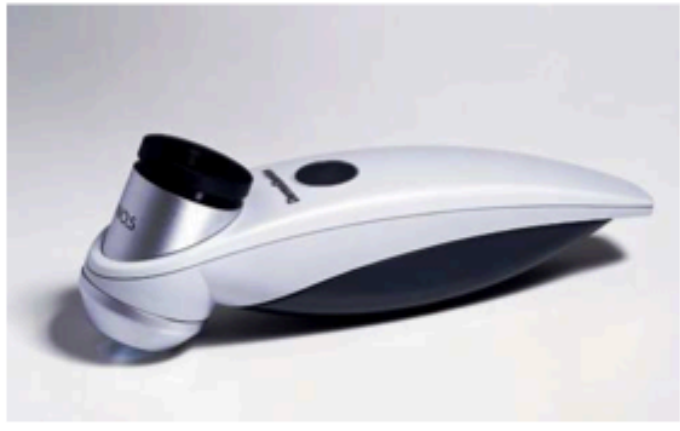

(d) DermLite(TM) DL100@

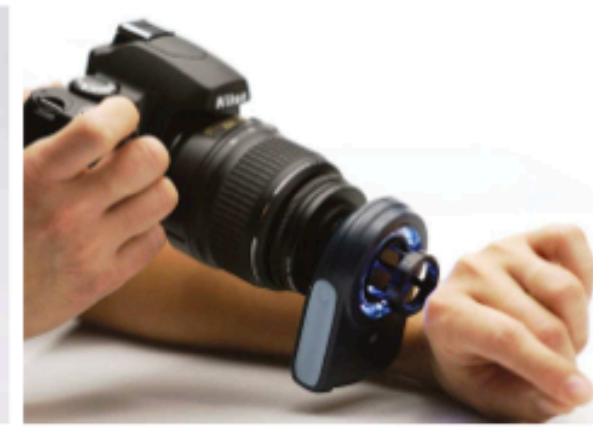

(e) DermLite II Pro@

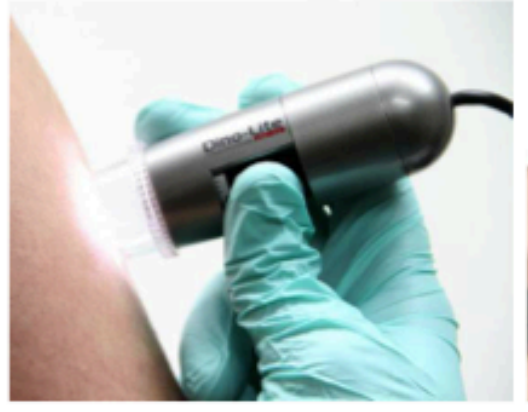

(f) DinoLite

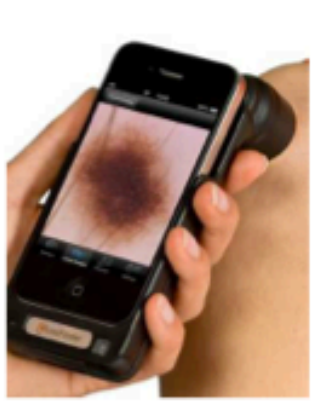

(g) Handyscope

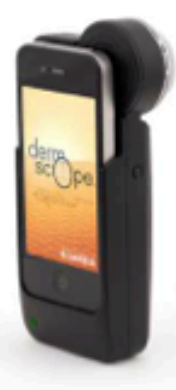

(h) DermScopeCanfield

Figure 2.3: Figures a, b, c, d, and e show analogue dermoscopes. DinoLite, Handyscope, and DermScope are modern digital dermoscopes shown in $\mathrm{f}, \mathrm{g}$, and h respectively [7]

\subsection{PREPROCESSING}

After gathering all the dermoscopy images, the next step is to do some preprocessing techniques to remove all the artifacts or sharpen the image using some filter techniques etc. For removing the lesion from the skin, we should first focus on doing some preprocessing on the skin to have smooth, clean and non-noisy images \& separating luminance information. 


\subsubsection{COLOR SPACE TRANSFORMATION}

After attached the dermoscopes to the digital camera and took a photo, sometimes the RGB images change to a scalar image because it has a simple computation. For this issue, we can use different methods like using the luminance transformation or applying the KL transformation etc. In some application the RGB is using and transfer to different color space to ensure approximate perceptual uniformity, achieving invariance to different imaging situations, $\&$ decreasing the number of channel [13].

\subsubsection{CONTRAST ENHANCEMENT}

Regarding to the pervious section, for detection of lesion border from the skin, we need to sharpen and illuminate the image. For contrast enhancement we can use the Delgado method to change the RGB image to a de-correlated color space and in this regard, the lesion and the skin are removed from each other with maximum rate [14]. More over, we should have illumination correction on the image cause some part of the image had shadow and bright space and also using high pass filter to sharpen the image by cancelling the low frequency noises.

\subsubsection{ARTIFACT REMOVAL}

Most of the dermoscopy images are contacting some artifacts such as hair, bubble and oil\& rulers that should remove from the images to have a better result in diagnosing of the melanoma. Figure 2.4 has shown three examples of dermoscopy images. One way to remove the noise and the artifacts is using some low pass filtering techniques to smooth the images Such as: Gaussian filter, Median filter etc. For using these filters we should pay attentions to some issues like Computational time, Mask size \& vector or scalar processing. The other way is to assign an specific method for each of the artifacts like for hair removal, we can use mathematical morphology and for the others, use an individual techniques. 


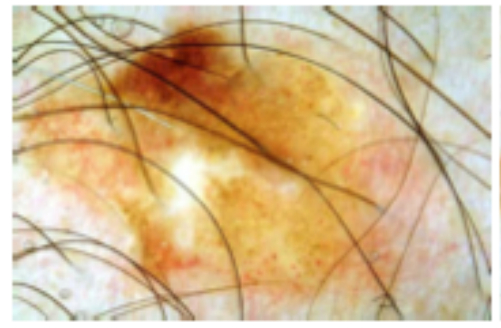

(a)

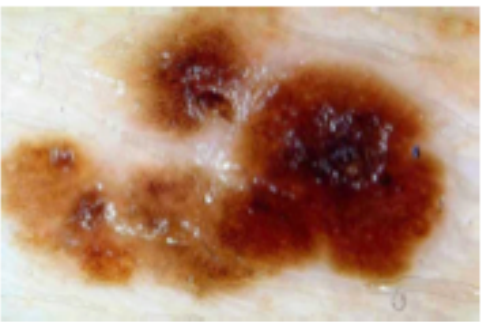

(b)

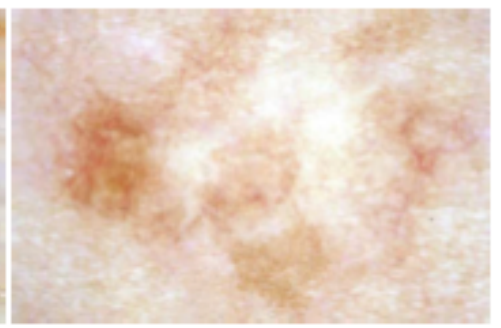

(c)

Figure 2.4: Three examples of dermoscopy images with hair (a), oil bubbles (b) and low-contrast (c) [7]

\subsection{SKIN LESION SEGMENTATION}

After gathering all the dermoscopy images and did some preprocessing techniques on the images, we should go through a segmentation part which as the most important part of our procedure. We should try to create new algorithm to have a high performance in separating the lesion from the skin cause this can affect on the other steps like feature extraction and at the end using some classification techniques to get a result with high accuracy. There are different segmentation algorithms, which have a good result in dermoscopy images that have been discussed in the pervious work section.

\subsubsection{DIFFERENT TYPES OF SEGMENTATION METHODS}

In segmentation part, the goal is to find the exact border of the lesion to remove the lesion from the skin and for the next steps, extract informative features from the lesion. There are different algorithms, which have been applied for the segmentation phase. Totally, we have different segmentation algorithm that can categorize them into 4 types. The more common segmentation algorithms, which can be found in different articles are such as: Global thresholding (GT), adaptive thresholding (AT), $\mathrm{k}$ means clustering (KM), fuzzy $\mathrm{C}$-means (FCM), expectation maximization (EM), statistical region merging (SRM), Active contour model (ACM), chan active contour model without edges (ACMWE), spectral clustering (SC) that will talk about it later in the pervious works section. The figure 2.5 shows 4 types of segmentation techniques such as [15]: 
1) Thresholding: This is pone of the simplest segmentation technique that changes the gray scale image into binary image.

2) Edge based segmentation: This method is using for a simple image and check if the pixels of an image is belonged to the lesion border or not!

3) Region based segmentation: In region method, the image is detached \& clustered into different regions that each of the regions has its own attributes.

4) Clustering based segmentation: This method is usually used for the gray level images that applied \& used for data with high dimension.

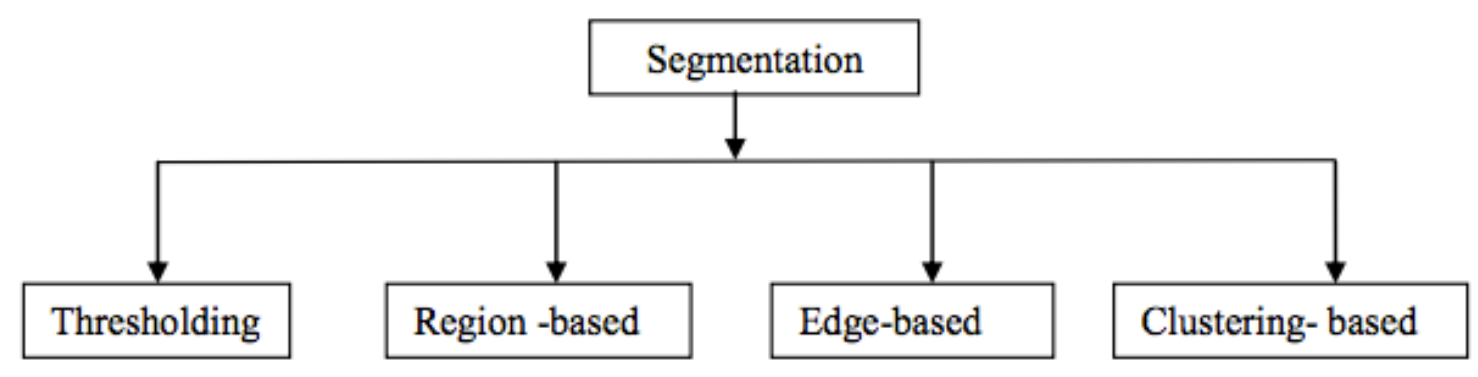

Figure 2.5: Different types of Segmentation [15]

\subsection{FEATURE EXTRACTION}

After segmentation part, we need to have a classification depends on our selected features. In the First section of this chapter, we have talked about general feature extraction like ABCD rule [16], 7 point checklist and menzies method. We have different feature extraction method which is using to characterize the skin lesion images, so its better to first detect and analysis the pigment network which is including of detection the hole and the network \& also the streak lines of the lesion and then we can have different feature extraction like lesion color texture features (LCT) [17] or STR feature that is a clinically inspired feature set [18]. 


\subsubsection{LCT FEATURES}

For extracting our features to use it as an input for a classifier we can focus on the lesion and color and texture of the lesion and extract these informative features from the streaks to have a better performance in our network. We can also have a combination of LCT with STR to have more features for our classifier about the lesion $[17,18]$.

\subsubsection{STR FEATURES}

The STR features is consist of 4 important features that is a clinically inspired feature set and its based on our streaks which are including: Structural features, Chromatic feature of streaks, Orientation features and geometric graph features which can use all STR features individually or a combination of STR with LCT as an input for our classifier. Figure 2.6 shows an absent, regular and irregular types of streaks which the third one shows a melanoma with irregular streaks [18].

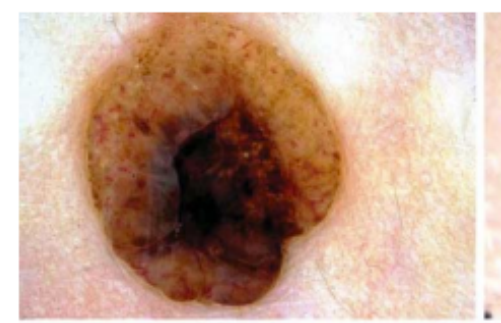

(a)

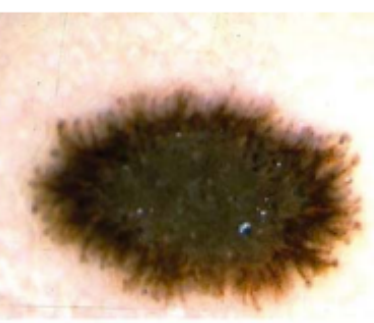

(b)

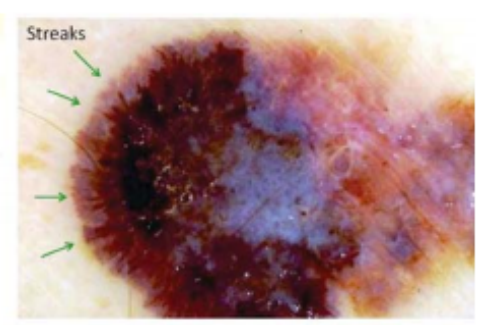

(c)

Figure 2.6: Examples of (a) Absent, (b) Regular and (c) Irregular streaks [18]

\subsection{CLASSIFICATION}

The next step after feature extraction and for the curve of dimensionality, using feature selection is Classification, which is the last phase to classify the 3 types of melanoma include absence, regular and irregular or having two class like absent and present. We have different classification methods such as artificial neural network classifier that can have classification methods with supervised or unsupervised learning such as MLP or SVM or clustering methods [19] like SOM or LVQ. We can also have a combination of different techniques like neural network with optimization method like genetic algorithm to have a better performance for our network. Now a 
day, Support vector machine (SVM) shows a good result in image processing field because of its kernel [20].

\subsection{MOBILE IMAGE ANALYSIS ALGORITHM TO DETECT MELANOMA}

These days, there are different kind of skin cancer application that using the smart phones to detect the melanoma like skin Vision or Dr Mole, Skincure etc. These smart phone applications can make it easier for the people that can capture their mole and most of the apps check the Asymmetry, Border, color, diameter and evolution of the mole and give us the risk of having the melanoma or not. There are 35 apps that's is available only for apple users [21]. Skincare is a new smart phone application that has two features. First of all, it has an intelligence \& real time alert, which can inform the user about the UV and the time of burning. Secondly, It can uses the dermoscopy images that a device can attach to your smart phone and you can take a picture of the mole and it uses an intelligence algorithm to do all the image processing like preprocessing, segmentation of the mole, feature extraction and at the end, do the classification to give us the result about cancerous or non cancerous of the mole. The figure 2.7 shows that the dematoscope devices attach to the iPhone and the user can take a picture of his mole [22]. These apps can save the time and money by taking a picture of your mole when you are at home to make sure about the situation of the mole. Some of the apps, which will give you, the result at the time has some problem that they are not more accurate and we can't rely on just their result. Its better that if the apps is suspicious about the mole, it can connected to a server to send the images to a specialist to check the mole and send back the result. In this way, we can say we have an accurate result using both the smart phone application with the opinion of the dermatologist. Some of the new apps have this facility to send the images for the specialist and have an accurate result like Skincure smart phone application. 


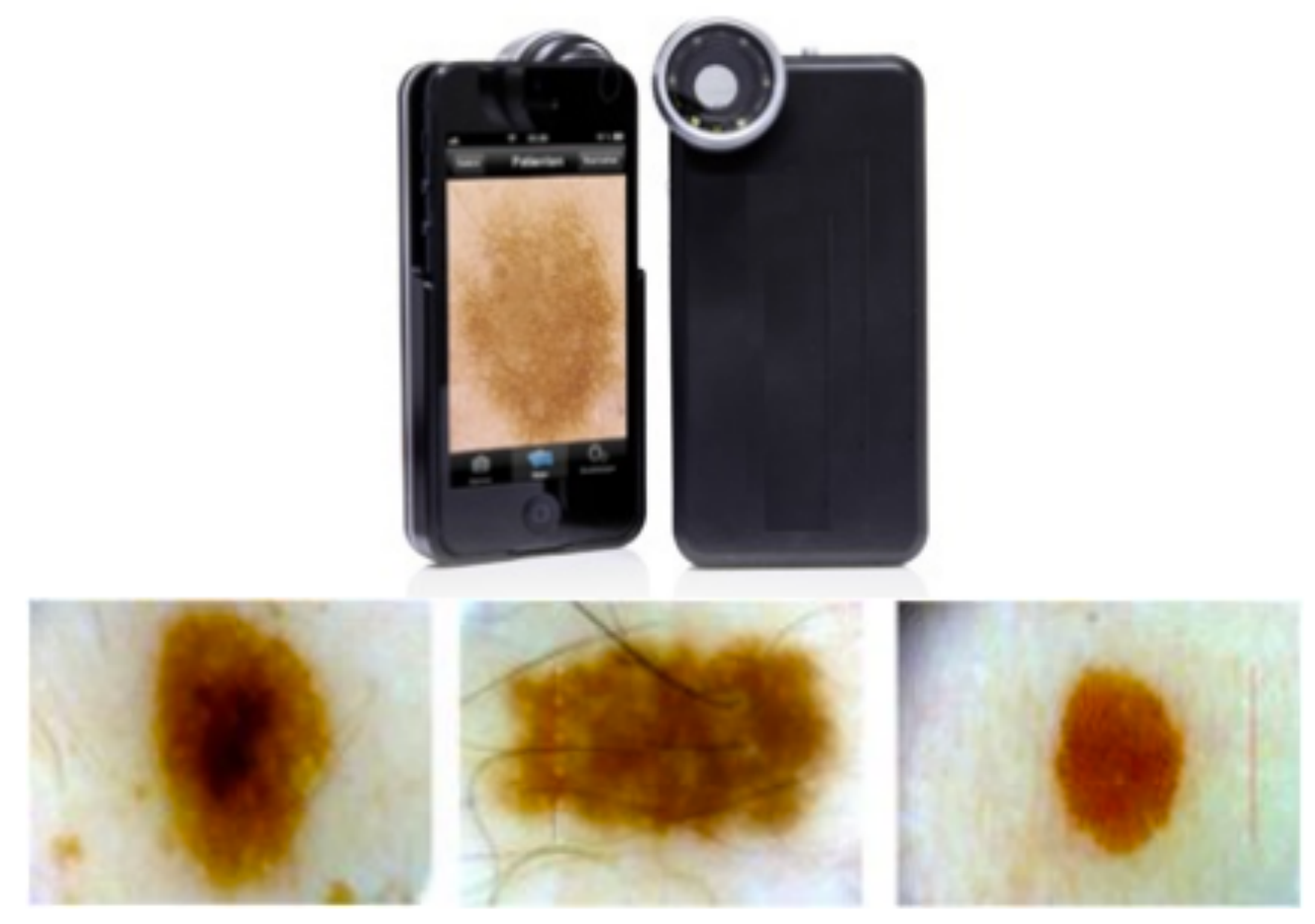

Figure 2.7: The dermatoscope device attached to the iPhone and sample of images captured using the device [22] 


\section{Chapter 3}

\section{PERVIOUS WORKS}

\subsection{MELANOMA \& NON-MELANOMA SKIN CANCER STATISTICS}

There are so many researches that have been done on skin cancer during the past of couple of years and they have gathered all the demographic data of incidence, mortality, survival, prevalence and potential years of life lost for melanoma skin cancer in men and female individually in table 3.1.An estimation shows that 6500 and 76,100 will experience of melanoma and non melanoma in 2014 at Canada respectively \& also 1,050 and 440 will die due to melanoma and non- melanoma respectively. The researches shows that the mortality of all cancers are $1.6 \%$ in men in 2009 and the incidence of all cancers in men has increased to $3.6 \%$ in men in 2010. We can also see the same trend of mortality and incidence in female that has increased $2 \%$ in a year. The survival of melanoma skin cancer was $85 \%$ and $92 \%$ in men and female between 2004 to 2008 respectively [3].

Table 3.1: Melanoma of the skin: Statistics at a glance in Canada [3]

\begin{tabular}{|c|c|c|c|c|}
\hline & \multicolumn{2}{|c|}{ Males } & \multicolumn{2}{|c|}{ Females } \\
\hline & Estimates* & Actual numbers ${ }^{\dagger}$ & Estimates* & Actual numbers ${ }^{\dagger}$ \\
\hline \multicolumn{5}{|l|}{ Incidence } \\
\hline Number of new cases & 3,500 & 2,965 & 3,000 & 2,535 \\
\hline Incidence rate $(\text { per } 100,000)^{\ddagger}$ & 15.9 & 14.7 & 13.0 & 11.9 \\
\hline$\%$ of all cancers & $3.6 \%$ & $3.4 \%$ & $3.2 \%$ & $3.0 \%$ \\
\hline \multicolumn{5}{|l|}{ Mortality } \\
\hline Number of deaths & 660 & 634 & 400 & 385 \\
\hline Death rate $(\text { per } 100,000)^{\ddagger}$ & 2.9 & 3.2 & 1.5 & 1.6 \\
\hline$\%$ of all cancers & $1.6 \%$ & $1.7 \%$ & $1.1 \%$ & $1.1 \%$ \\
\hline \multicolumn{5}{|l|}{ Survival } \\
\hline Five-year relative survival ratio (estimates for 2004-2008) & $85 \%$ & - & $92 \%$ & - \\
\hline \multicolumn{5}{|l|}{ Prevalence } \\
\hline 10-year person-based prevalence (Jan. 1, 2009) & - & 19,895 & - & 19,600 \\
\hline Potential years of life lost (for 2009) & - & 11,800 & - & 8,000 \\
\hline
\end{tabular}


The non-melanoma skin cancer statistics shows that the incidence rate for basal cell carcinoma (BCC) and squamous cell carcinoma (SCC) was 120.7 and 39.6 in 2011 respectively. The 2011 and 2010 information was for Alberta and Quebec respectively. The statistics show that the five years survival ratio is 101\% and 95\% for BCC and SCC between 2007 and 2011 respectively. Most of the researches were on melanoma and non-melanoma skin cancer, which are the two important types of skin cancer. In Non-melanoma skin cancer (NMSC), the SCC is tending to be more aggressive than BCC. The Table 3.2 shows the incidence for non-melanoma skin cancer for selected province and years for BCC and SCC and also the five-year relative survival ratio for both sexes combined between 2007 to 2011 [3].

Table 3.2: Non-melanoma skin cancers (BCC and SCC): Statistics at a glance based on selected provinces [3]

\begin{tabular}{l|c|c}
\multirow{2}{*}{} & \multicolumn{2}{|c}{ Both sexes combined } \\
\cline { 2 - 3 } & BCC & SCC \\
\hline Incidence (for selected provinces and years) & & \\
\hline Number of new cases* & 13,655 & 4,015 \\
\hline Incidence rate (per 100,000) & & 39.6 \\
\hline Survival & 120.7 & \\
\hline Five-year relative survival ratio (estimates for 2007-2011) & & $95 \%$ \\
\hline
\end{tabular}

\subsubsection{TRENDS IN INCIDENCE AND MORTALITY}

The melanoma skin cancer is including the $3 \%$ of all the new cancer and it has a place of top 10 cancers in Canada, so because most of the cases can be diagnosed so soon, we can reduce this amount to less than that in the near future. The figure 3.1 shows the age standardized incidence \& mortality rates of melanoma skin cancer by sex between 1986 to 2010 and 1986 to 2009 respectively. If we look at the figure and compare the incidence and mortality rate during the 25 years, its more obvious that the incidence in both male and female has increase very well than the mortality in both sex [3]. 


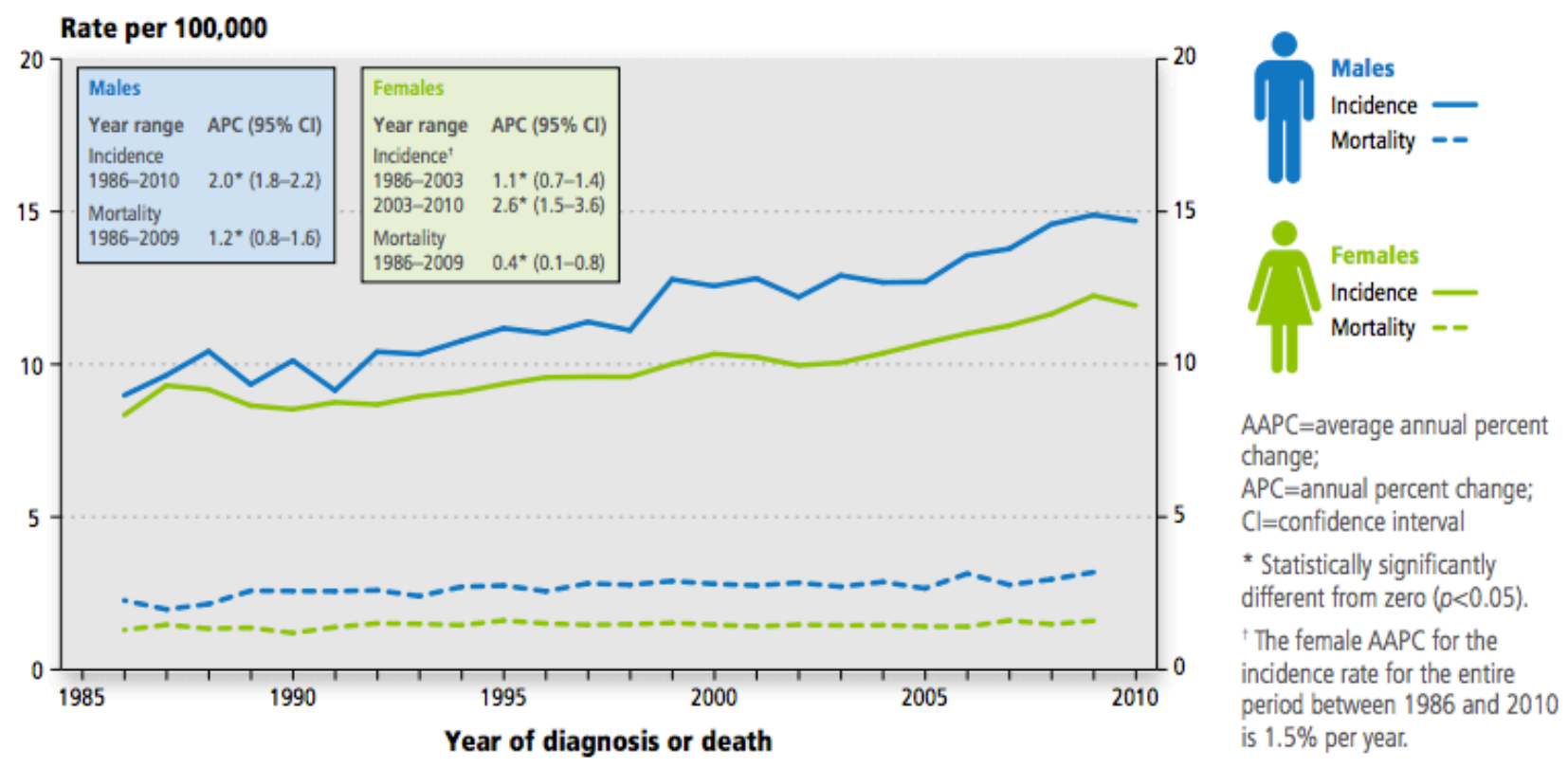

Figure 3.1: Age-standardized incidence (1986-2010) and mortality (1986-2009) rates of melanoma of the skin by sex, Canada [3]

\subsection{PRE-PROCESSING}

To have a better segmentation on skin lesion, we should do some pre-processing on the image. Most of the dermoscopy images have some artifacts, noise that should use different techniques to remove all the oil, bubble, hair, noise and also sharpening the image using the high pass filter.

\subsubsection{REMOVAL OF ARTIFACTS}

As we mentioned in the pervious chapters, the dermoscopy images contain different artifacts like oil, bubble, noise, hair and illumination etc., so we need an automated techniques to remove all these artifacts from the dermoscopy images to have a better segmentation of lesion border. There are some methods that are using to remove the artifacts and sharpening the image with ignoring the low pass frequencies. Abbas et al. [23] had created a method for removing the hairs in dermoscopy images. They had 3 classes of hair removal methods such as: exemplar based methods [23,24], in painting by non-linear PDE based diffusion algorithms [25] \& linear interpolation techniques $[7,26,27]$. In some articles, they also use different filtering techniques for sharpening the image or removing the noise. 


\subsection{SKIN LESION SEGMENTATION}

The Goal of the image segmentation is to have the best separation of the lesion from the skin to extract the informative features from the lesion. We can define the lesion segmentation as a set $\mathrm{L}$ $=\{$ 'lesion', 'background' $\}$ to the pixels in the image, so we can check the probability of belonging every pixel to the lesion or background [28]. During the past couple of years, there are so many articles, which have been worked on the segmentation part for separating the pigmented skin lesion from the background of an image [29,30,31,32,33,34,35,36,37,38,39].

At the first, $\mathrm{Xu}$ et al. [36] created an automatic segmentation algorithm for separating the lesion from the skin in clinical images. Then, Li et al. [34] showed that adding 3D depth information to RGB color images could improve segmentation of pigmented and non-pigmented skin lesion. We have so many segmentation methods, which used different techniques like active contours, Fuzzy logic, region growing, image thresholding, genetic algorithm, random walker etc. Or had a combination of these algorithms and received a good result in segmentation part. In image thresholding, after we choosing the threshold level, then we can assign the pixel with higher intensity into lesion class and the other ones with lower intensity into image background [40]. Lee and collaborators [41] created a methodology based on region growing method for feature extraction from ultrasound images.

The new method had worked on fuzzy logic to introduce a suitable level to determine that each pixel is belonging to the skin lesion or the background of the image with specific weight between 0 to 1 [42]. Furthermore, the fuzzy C-means algorithm (FCM) is applied for the segmentation of the skin lesion using fuzzy logic rules [43,44]. The other articles had worked on Genetic algorithm for the segmentation part [45-47] cause GA is try to use some operations like crossover and mutation to find the best answer between our initial population using the fitness function.

Celebi et al. [48, 49] presented a rapid and unsupervised method based on the statistical regionmerging algorithm (SRM). Before that, Celebi et al. in [50] had decreased the dermoscopic image to 20 different color groups, and checked each pixel to see which color groups it belongs to. In another attempt, Iyatomi et al. introduced a DTEA segmentation algorithm [51] that is trying to find high frequency parts and then thresholding with Otsu technique [52]. 
In another paper, an automated method is presented using a combination of multiple thresholding techniques [53]. The other methods that have been used for the segmentation parts were wavelet transform where the approximation coefficients are displaced to be effective in segmentation. In this article, some novel approaches like W-FCM, W- CPSFCM, and WK-Means, have been employed in segmentation part [54]. The other methods for segmentation part was initializing the random walker with seed points [55] \& A hybrid region-based active contour model with intensity inhomogeneity that was more effective \& robust than the Lankton method (LRBAC) \& Chan-Vese active contour model respectively [56]. Emre et al. [57] has presented two clustering algorithms: DBSCAN and STING-based segmentation algorithm that the first one had a high performance than the second one (STING). 


\section{Chapter 4}

\section{DERMOSCOPIC IMAGE SEGMENTATION}

\subsection{IMAGE DE-NOISING \& ENHANCEMENT}

Regarding to pervious Chapter, we should do some pre-processing techniques on the image such as: remove some artifacts like oil, bubble, hair, removing the noise or sharpening the image by using high pass filter etc. For detecting and removing the hair, we can also use several Morphological operators and threholding [62]. For the implementation of our algorithm in the segmentation part, I have used a pre_process function in MATLAB, which is first a margin is calculated for the input image and then we assign the skin color to the edge and corner of the image, by using the calculated margin and value of the skin color. By doing this technique, we can have a normal background when we want to detect or segment the lesion from the skin.

\subsection{EXISTING SEGMENTATION METHODS}

Image segmentation is an important part, which can categorize each image into some regions base on some characteristics like texture, color, shape etc. Totally, we have two type of segmentation named feature domain and image domain. In feature domain, we are searching to find a tiny cluster between the features. It's like that some features is measured for each pixel like color, texture, shape, intensity etc. \& we managed it in feature vector. Finally, we use clustering or thresholding method for segmentation. In image domain, we check the common features between the pixels and belonging of each pixel to a region. For classification, can use region method or boundary method to classify the pixels [58]. They are too many segmentation methods but I have focused on the most common segmentation algorithms such as: gradient vector flow snakes, automatic thresholding, region growing, k-means, mean-shift, and watershed [59]. Thresholding is a simple technique, which is using for the segmentation part of the image. We obtain a threshold and categorize all the pixels into two groups based on having the amount more or less than a threshold. We can assume the pixels less than a threshold is zero and the 
pixels more than that is 1 .

At the end, all the pixel that has an intensity of 1 is belonging to the lesion and the entire pixels that are zero is belonging to the background [60]. This techniques is so simple and fast but has some disadvantages like its completely depends on the threshold $\&$ also the threshold can't show the spatial information of the image. We have two automated thresholding such as triangle and Otsu's method that the second one is more used in different articles. Figure 4.1 has categorized all the segmentation methods and we tried to focus more on the region and contour based method or using a hybrid version of the algorithm to get the best result [61].

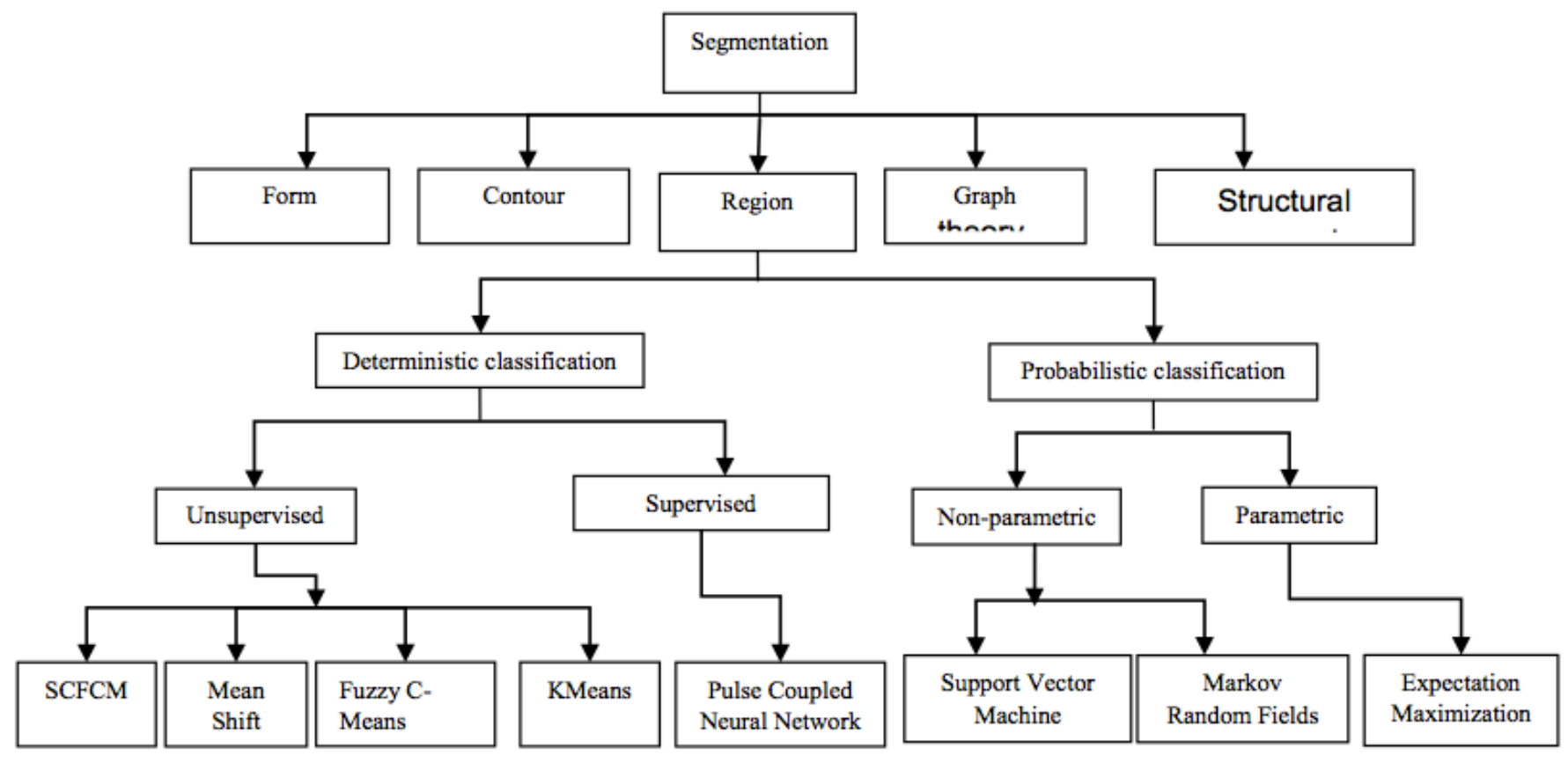

Figure 4.1: Region-based segmentation methods [68]

\subsection{PROPOSED METHODS}

Segmentation part has an important affect on the accuracy of the system but finding the appropriate segmentation method is so difficult cause we have different skin types and the color, texture and shape of the lesion. As we said we had three different types of segmentation methods like thresholding, edge based and region base for dermoscopy images. Creating a new or hybrid 
algorithm for segmentation of the skin lesion is an essential part for detection of skin cancer, so I have focused on implementing different segmentation algorithms in different areas like support vector machine for lesion detection in MRI images [63], using Self organizing map (SOM) for skin image segmentation [64] or brain MRI image segmentation [65]. The other proposed methods in this project are Using a hybrid segmentation algorithms to have a better performance such as: Breast MRI Tumour Segmentation Using Modified Automatic seeded region Growing based on particle swarm optimization image clustering [66] or Generalized rough fuzzy c-means algorithm for brain MR image segmentation [67]. There are so many segmentation algorithms in different articles but I have chosen the hybrid version of different common \& famous algorithms, which just have been used for the segmentation of brain and breast MRI tumour images.

\subsubsection{SEEDED REGION GROWING USING PARTICLE SWARM OPTIMIZATION}

This is a Hybrid method of Automated seed region growing and particle swarm optimization image clustering for the segmentation of skin lesion [66]. This article is created a hybrid method for the Magnetic Resonance Imaging (MRI) breast tumour segmentation. In this project, first, we do some prepossessing on the image. Then, we have chosen Seed region growing (SRG) cause its simple, rapid and too robust [69]. We have so many optimization methods like Genetic algorithm, ant-colony, and particle warm optimization etc. but in this project, we also have chosen PSO clustering cause it has a better performance compares to the other clustering algorithm like Fuzzy C mean, K-means and genetic algorithm [70-72].

\subsubsection{Seeded Region Growing (SRG)}

First, we use the seed and threshold process based on Particle swarm clustering \& then we have applied the seed region growing to the system, which has shown in figure 5.2.The SRG [73] algorithm is starting from a see point in the center and we compare it to its eight neighbours using threshold value to classify them based on intensity and then again each of the eight neighbours act like a initial see point and will compare with their neighbours. We should choose two factors manually are SRG such as the initial seed pixel and the threshold value. 


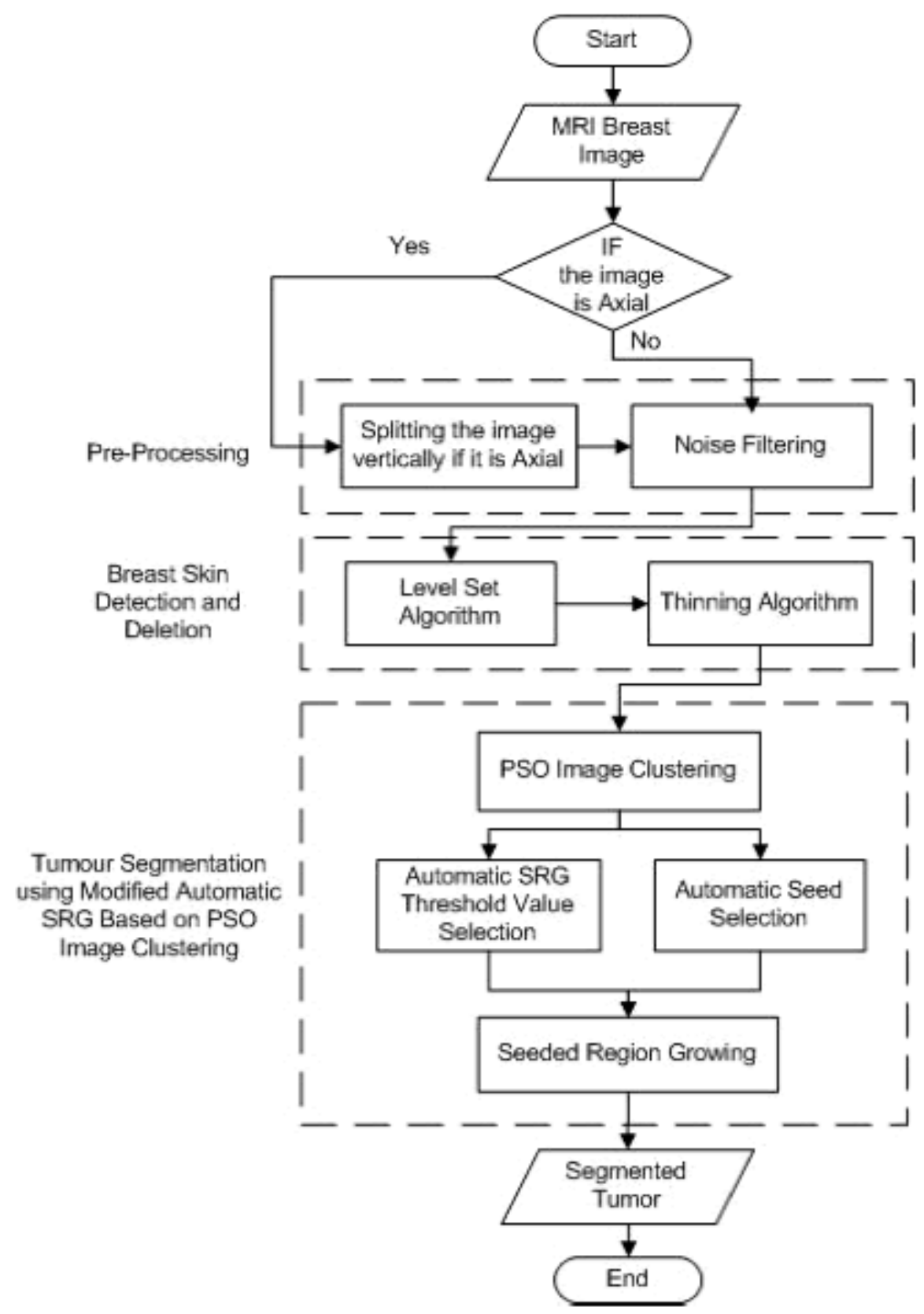

Figure 4.2: Methodology Flowchart [66]

\subsubsection{Particle Swarm Optimization (PSO) Image Clustering}

Particle swarm optimization is a proper algorithm for image segmentation comparing into other clustering methods and it has been used in different segmentation area [74-78]. First, we use an integration of level set active contour algorithm with morphological thinning algorithm to remove the lesion from the skin [89]. In PSO, we create different clusters based on having 
different intensity. The procedure of automatic SRG seed selection, which has shown in figures 4.3 are:

1 (a): We use Particle swarm optimization on dermatoscopy images

2 (a): Then rating and classify the clusters based on their intensity value.

3 (b): Choosing the highest PSO cluster region and remove the entire clusters.

4 (c): The location of the center pixel in the zone that is the initial seed is selected automatically (the red point in figure 4.3) \& it will compare with its neighbours

5 (d): Using the threshold to compare the seed with its eight neighbours and classify them based on intensity (The blue region)

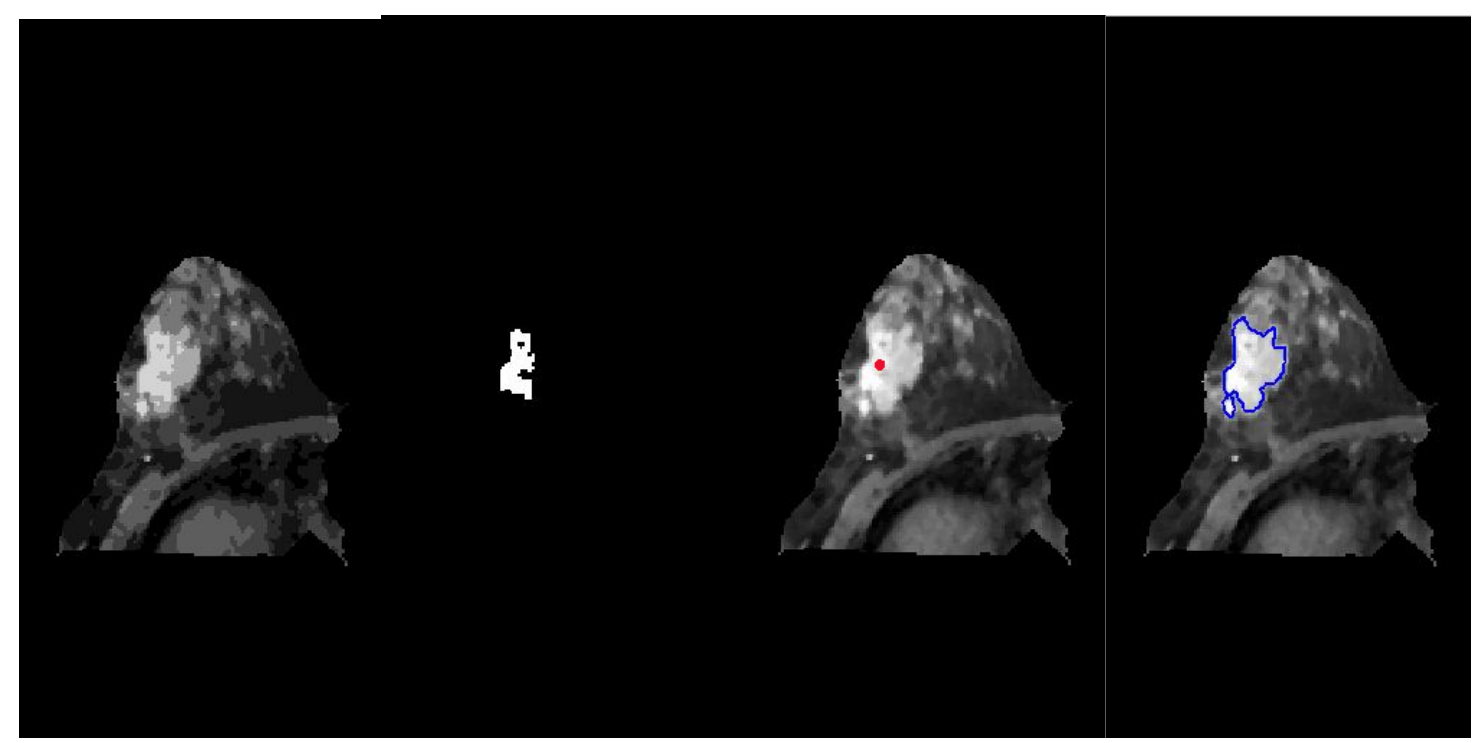

(a)

(b)

(c)

(d)

Figure 4.3:The Proposed Automatic SRG Initial Seed Selection [66] 


\subsubsection{GENERALIZED ROUGH FUZZY C-MEANS ALGORITHM}

The other proposed method is implementing the rough fuzzy c-means algorithm, which has been used for brain MR image segmentation for our dermatoscopy images [67,79]. In this method, we combined the rough c-mean and fuzzy c-mean techniques for the segmentation of skin lesion. Applying a hybrid version of Fuzzy set and rough set is so useful in clustering problems in medical image segmentations. The proposed c-means combine the idea of fuzzy membership of fuzzy sets and lower and upper approximations of rough sets into c-means algorithm [80]. To determine the rough fuzzy region, we assign three rough fuzzy regions to each cluster based on two thresholds. We should calculated the belonging of each pixel to each cluster and then we should see that the pixel is belonging to which one of the clusters more than the others and the center of that cluster should be updated. The figure 4.4 shows how to determine the rough fuzzy regions .All the pixels who are in the positive regions has the membership of 1 and the entire pixel belonging to the negative region has the member ship of zero. Equation 2 shows the simplified objective functions and the weighting parameter.

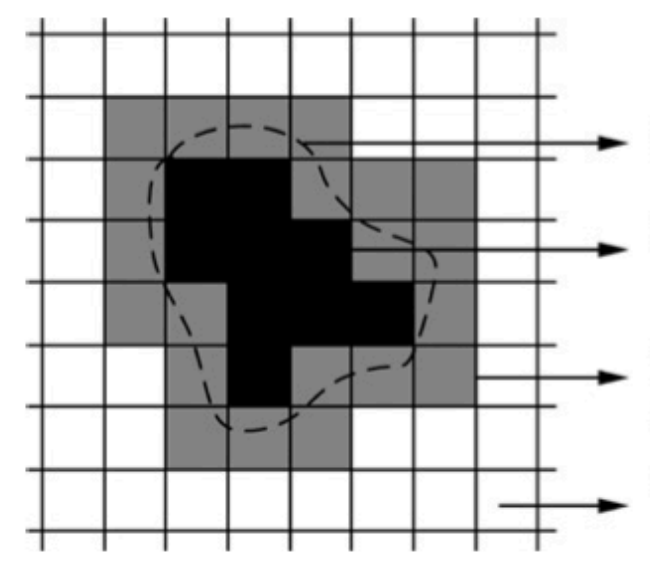

uij: membership value

Cluster vi Positive Region $\mathrm{P}(\mathrm{vi}) \quad \mathrm{u}_{\mathrm{ij}}=1$ Boundary Region $\mathrm{B}\left(\mathrm{v}_{\mathrm{i}}\right) \quad \mathrm{u}_{\mathrm{ij}}=[0,1]$ Negative Region $\mathrm{N}\left(\mathrm{vi}_{\mathrm{i}}\right) \quad \mathrm{u}_{\mathrm{ij}}=0$

Figure 4.4: Illustration of three rough-fuzzy regions [67]

$$
J_{G R F C M}=\lambda \sum_{c=1}^{C} \sum_{i \in P\left(v_{c}\right)}\left\|I_{i}-b_{i} v_{c}\right\|^{2}+(1-\lambda) \sum_{c=1}^{C} \sum_{i \in B\left(v_{c}\right)} u_{c i}^{m}\left\|I_{i}-b_{i} v_{c}\right\|^{2}
$$


After the clustering the image and the lesion is detected, we used the normal-segmented image function in MATLAB to remove the artifacts, which are less than 50 pixels from the background.

\subsubsection{SUPPORT VECTOR MACHINES (SVM)}

For segmentation, we can use different supervised classifier like linear discriminant analysis (LDA), Quadratic discriminant analysis (QDA) or support vector machine (SVM) etc., which SVM is a supervised machine learning that has been used for diagnosis of melanoma in the past [81]. We prefer to use SVM cause it's a non-parametric technique that can manage the huge data. The idea of SVM is to have a non-linear mapping of data into high dimensional feature space and the most important part is to make a decision to choose which kind of kernel based on the distribution of our information. We have different kernel methods such as: linear, polynomial and radial basis function that RBF is a good kernel for Gaussian distribution [63]. In this scenario, we can have one class, two classes or multiple class of SVM, which in two classes, we have the positive, and negative class belongs to lesion and non-lesion data respectively. The decision boundary has been made by the training samples. The figure 4.5 shows the decision boundary that two classes is trained \& has shown by 'o' and '+' \& for one class of SVM, it has shown by just 'o' [82].

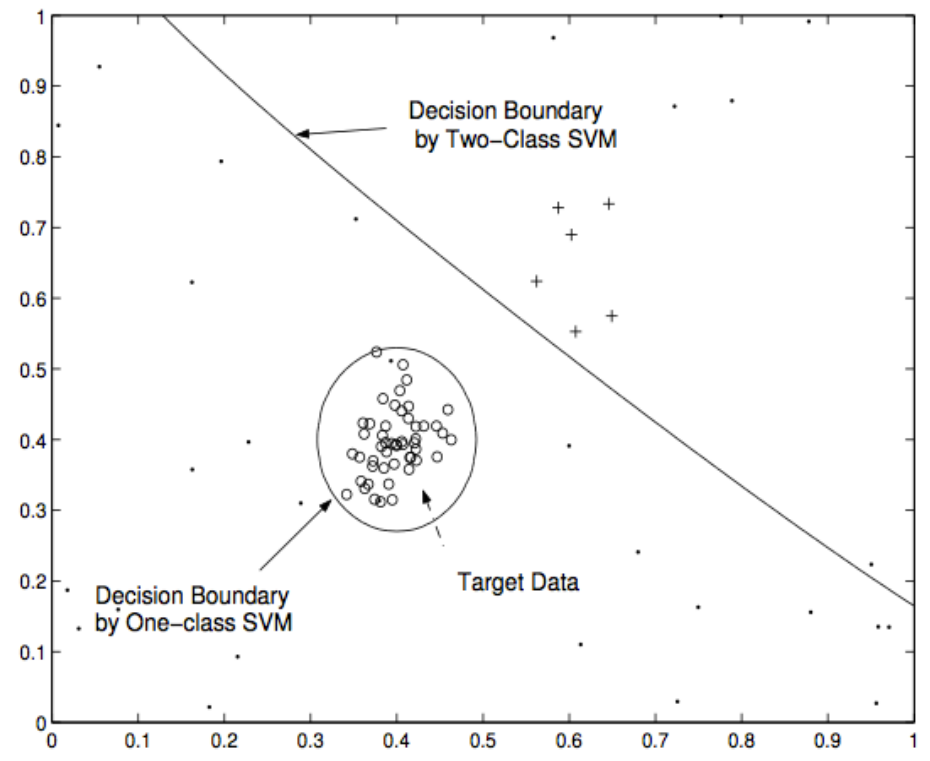

Figure 4.5: Two-class SVM is trained on the samples indicated by 'o' and ' + ' \& One-class SVM is trained only on the samples indicated by 'o' [82] 


\subsubsection{SELF-ORGANIZING MAP (SOM)}

Self-organizing map is an unsupervised machine learning that has been used for the segmentation of medical images [83,84,85]. Two things are important in SOM such as: training the network and mapping [85] \& as you can see in Figure 4.6,it has two layer. The first layer is including the inputs and the second layer is the outputs $\&$ each node will connect to the output with a specific weight [86]. First, to apply SOM on the image, we should change the RGB image into two-dimensional array. Then we should train our SOM network by the training data. We use the Normal color function and we assume that each pixel of an RGB image that has lowest and highest value of each color (red, green and blue) are including the skin lesion. After we calculated the skin color values and the margin, the next step is to transfer the input image into small 10* 10 pixel image \& regarding to a threshold that has been assumed for the lesion, we obtain that which one these small images are closed to the lesion. If in each small image, more than $30 \%$ is belonging to the lesion, then we can say it's close to the lesion $\&$ then we update the coordinates that we assumed for the region of the lesion. More over, we also use the Sobel operator in edge detection to extract the lesion border from the image and regarding to the border, we draw a yellow circle for the region of the lesion.

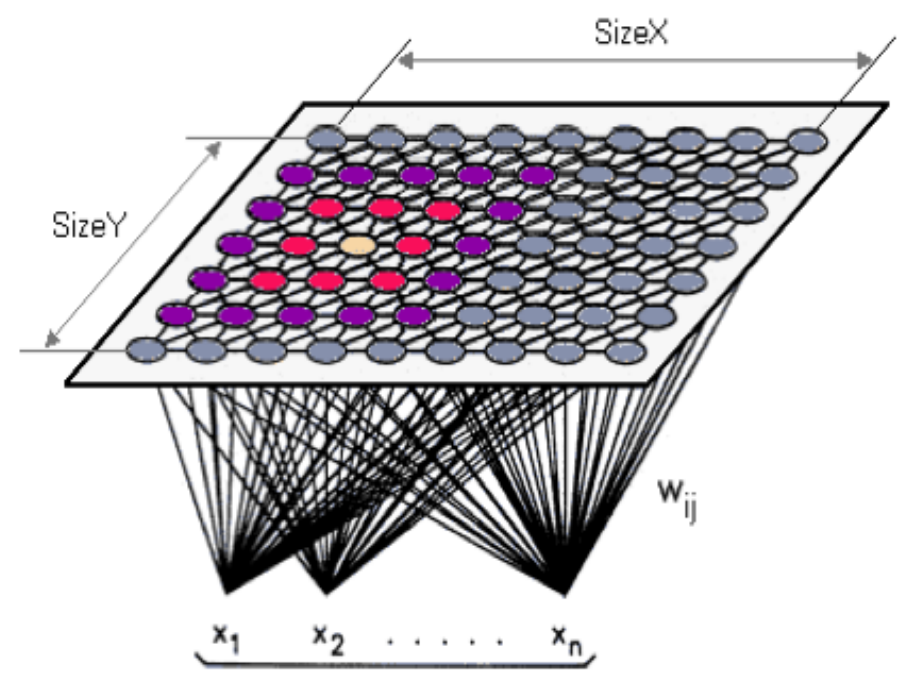

Figure 4.6: Mapping of feature vector to the output [83] 


\section{Chapter 5}

\section{SEGMENTATION RESULT AND DISCUSSION}

\subsection{DATASET}

Gathering a high quality of dermoscopic images for the project was a difficult task and after Cooperating with Meta Optima Company for the segmentation phase of the project, we could gather 108 epiluminescence microscopy (ELM) image, which is a non-invasive technique that, by employing the optical phenomenon of oil immersion can have a better detection of pigmented skin lesions \& diagnosis of early stage of malignant melanoma [87]. We also could get 200 Incontinence-associated dermatitis (AID) images. The second dataset is gathered from the website (Derm101.com) that could access to 132 dermoscopy images for using in segmentation part.

We also have gathered the ELM \& IAD Database with Mask that is calculated by the specialist or radiologist as a ground truth manually. The mask can help us to compare our segmentation result with the ground truth and check the performance of our methods. Figure 5.1 shows an original ELM image and it's mask evaluation the performance.
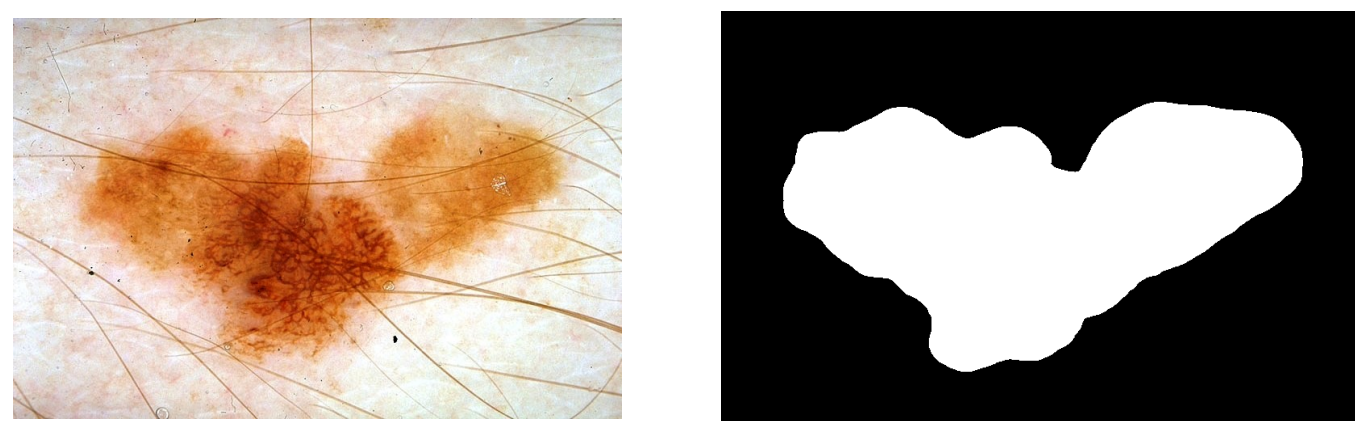

Figure 5.1: An epiluminescence microscopy (ELM) image is on the left and its mask is on the right 


\subsection{PERFORMANCE MEASURES}

To show the performance evaluation of our segmentation method, we use some famous performance measure that is more common in different articles to evaluate the accuracy, sensitivity and specificity of the segmentation techniques [88].

$$
\text { Accuracy }=\frac{\#(T P)+\#(T N)}{\#(T P)+\#(T N)+\#(F P)+\#(F N)}
$$

Sensitivity $=\frac{\#(T P)}{\#(T P)+\#(F N)}$

$$
\text { Specificity }=\frac{\#(T N)}{\#(T N)+\#(F P)}
$$

\subsection{EXPERIMENTAL RESULTS}

All the segmentation techniques have implemented in MATLAB_R2014b and use both group of dataset for the segmentation part. At the end, all the automatic segmentation result has been compared with the ground truth to check the accuracy and performance of our method. All the experimental result includes the figures of the segmentation procedure and comparison table of the methods have shown in the following section.

\subsubsection{EVALUATION OF SEGMENTATION ALGORITHMS}

In this part, we have shown all the result of the segmentation techniques such as: Automatic Seeded Region Growing using Particle swarm optimization, rough fuzzy c-mean algorithm, Support vector machine (SVM) and self organizing map (SOM) for segmenting the skin lesion from the background. The first method is using PSO for the clustering the image and then based on seed region growing. The figure 5.2 has shown the level set active contour parameter that should be set manually first \& also the level set evolution at 189 iteration for removing the skin from the lesion. 
For the level set algorithm, we initialized the Scale parameter in Gaussian kernel \& the parameter in the definition of smoothed Dirac function as 1.5 , the coefficient of the internal energy term p equal 0.04 , the coefficient of the weighted length $\&$ area term $5 \& 1.5$ respectively $\&$ the number of iteration equal 500 .
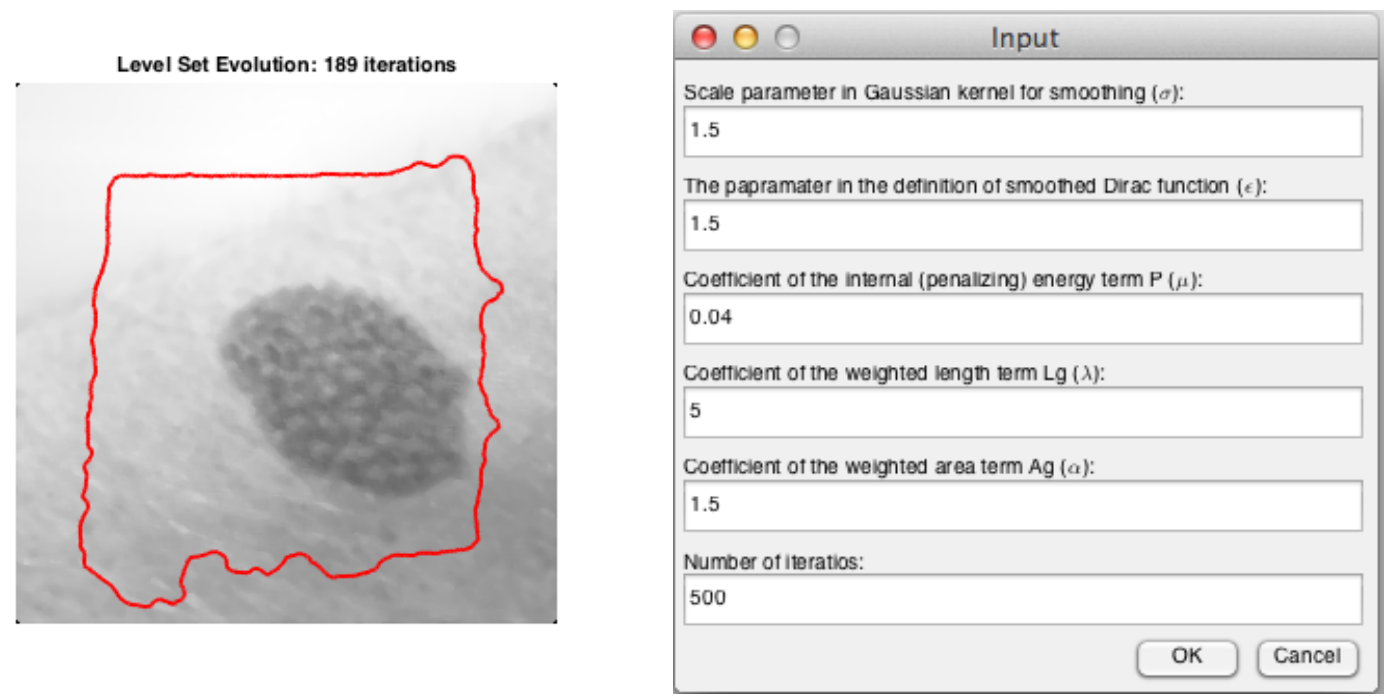

Figure 5.2: The left one shows the level set evolution of PSO clustering after 189 iteration and the right one shows the initial parameters for the clustering

The Figure 5.3 shows all the procedure to detect the skin lesion using automated see region growing using particle swarm optimization. We should transfer the RGB image into grayscale and start to draw a shape for skin lesion with level set contour algorithm using morphological operations and the last image show the result of using all the techniques to calculated the lesion region. 


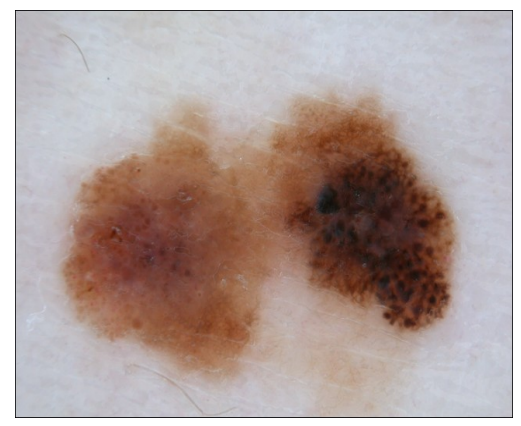

(a)

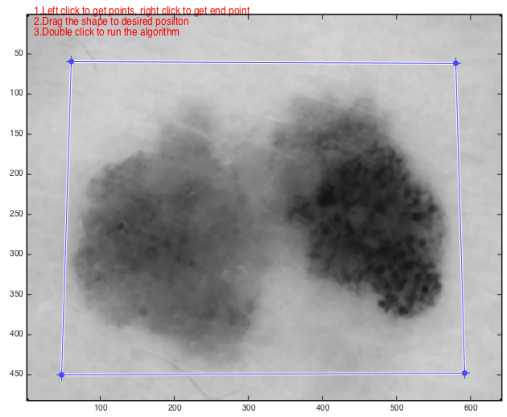

(c)

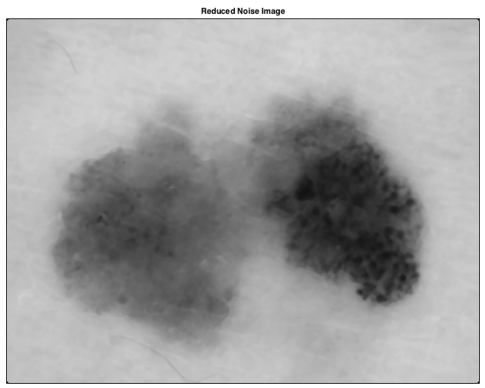

(b)

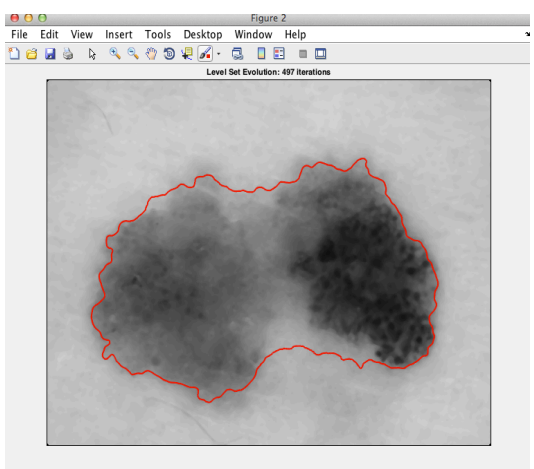

(d)

Figure 5.3: Original image(a), grayscale image(b), draw the shape to desired position(c), segmentation result (d)

The second algorithm for the segmentation part is rough fuzzy c-means algorithm, which is using a hybrid version of some algorithms to have a better performance. The figure 5.4 has shown the original image, segmented image, the edge detection technique on the segmented images (lesion) and the output that the lesion is detected completely. The figure 5.5 is the same as figure 5.4 for another dermoscopy image to compare the rough fuzzy c-means technique using two different images. It is obvious that, our segmentation method could segment the epiluminescence microscopy (ELM) image better than the dermoscopy image. 

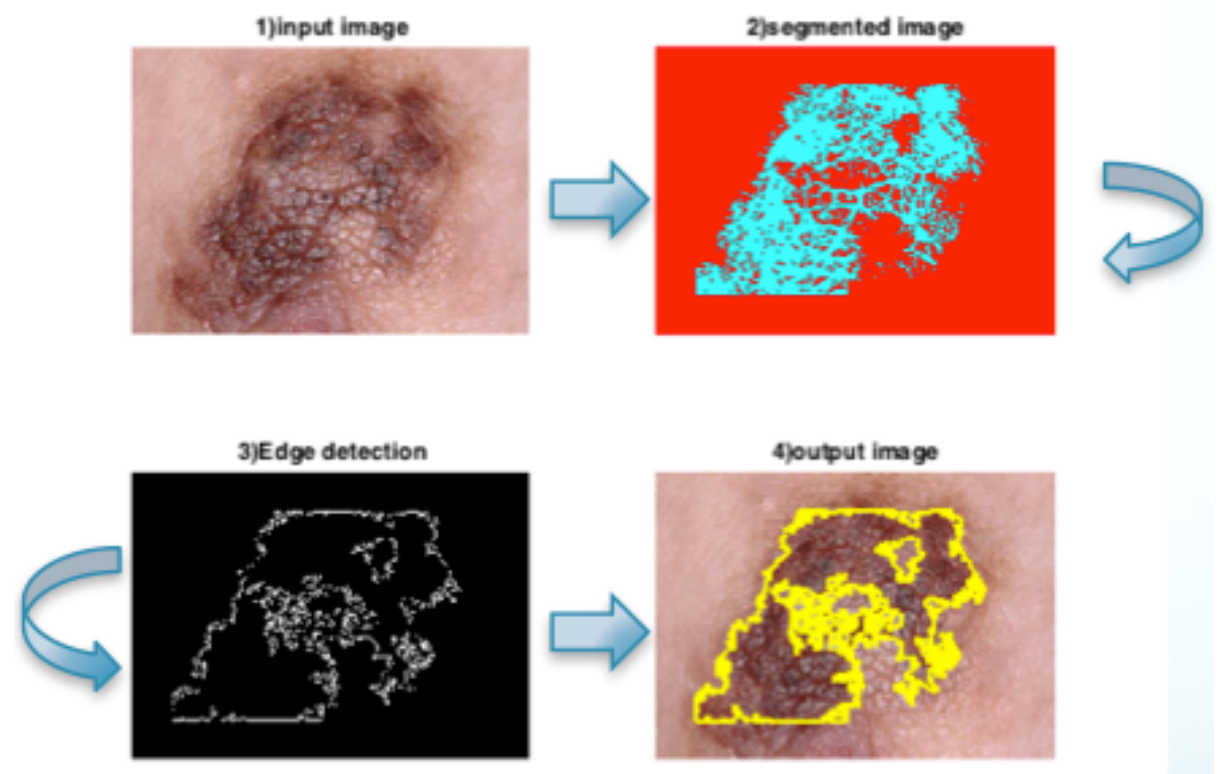

Figure 5.4: The procedure of skin lesion segmentation using rough fuzzy c-means algorithm for one ELM image of the database
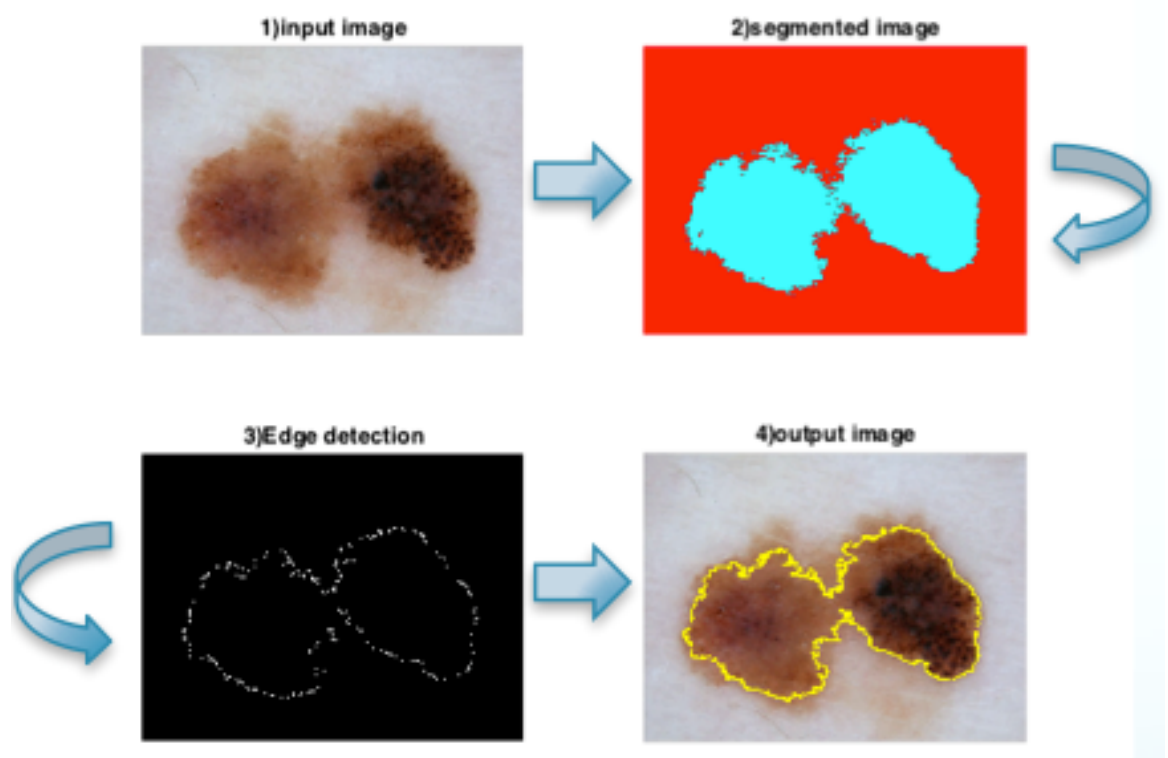

Figure 5.5: The procedure of skin lesion segmentation using rough fuzzy c-means algorithm for one Dermoscopy image of the database 
The third segmentation method was Self-organizing map (SOM), which is an unsupervised machine learning method. The figure 5.6 has shown the SOM procedure including the original image, segmented image, the edge detection on segmented image and the result of SOM network in skin lesion segmentation. We use the neural network toolbox in MATLB and the figure also shows that the network is trained after 200 iteration and then stopped.
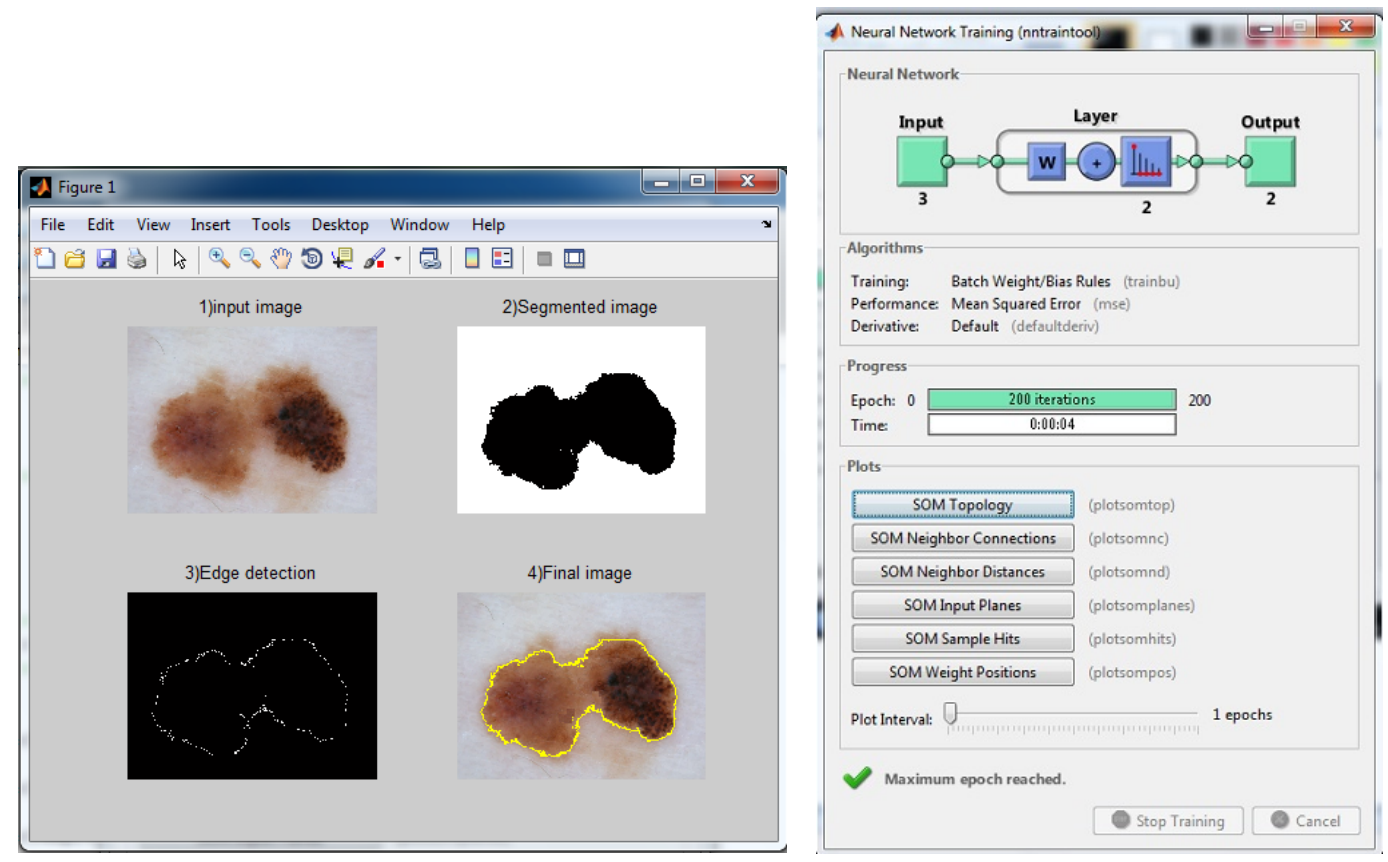

Figure 5.6: The procedure of SOM segmentation is on the left and the training of the SOM network after 200 iteration is on the right

The last segmentation method is support vector machine (SVM), which is more common to use in different segmentation areas. The figure 5.7 shows the support vector machine procedure for segmenting of skin lesion in two different dermoscopy images. Both of the figures show the original image, segmented image, edge detection method on the segmented image and the final result of the skin lesion segmentation. Both figures show that the SVM in the lower figure could segment the skin lesion better than the upper one. 

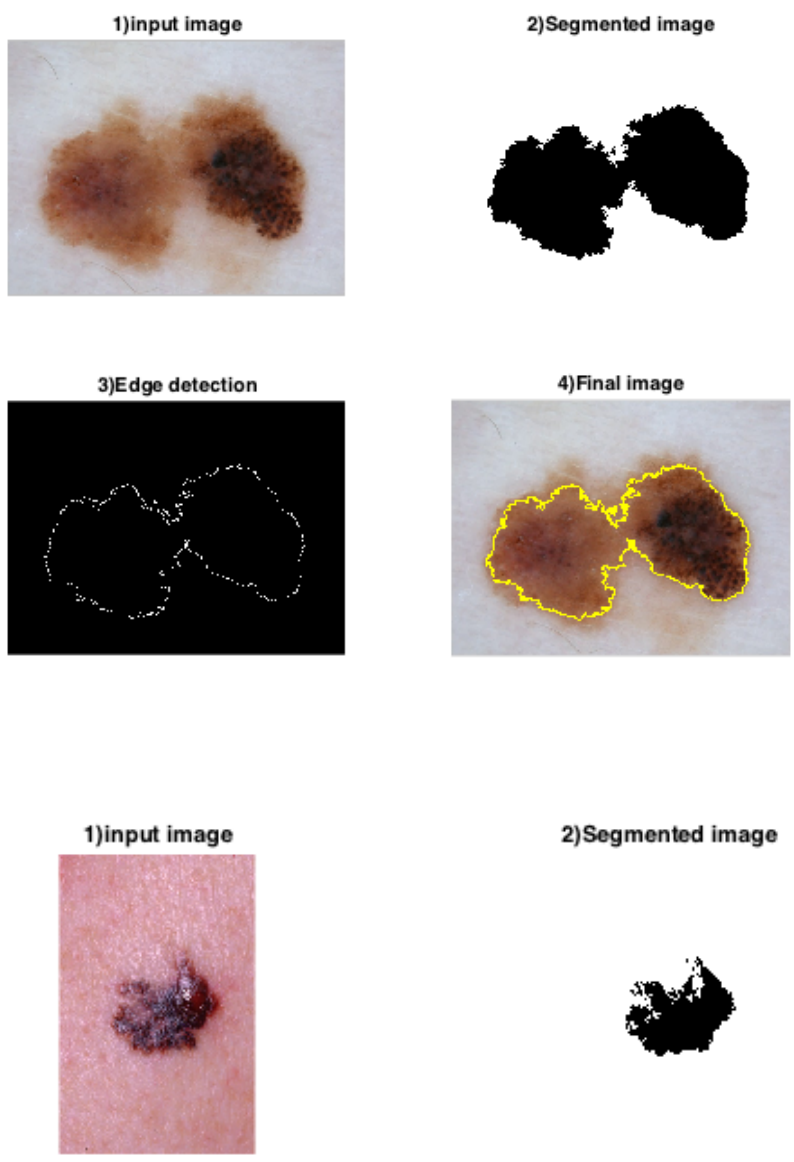

2)Segmented image
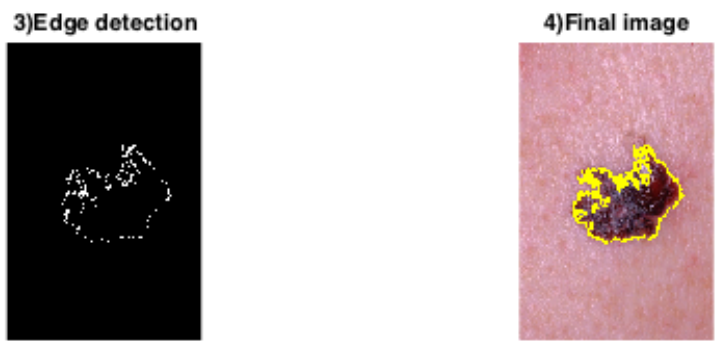

Figure 5.7: The support vector machine procedure for segmenting the skin lesion in two different demoscopy images. 


\subsubsection{COMPARISON OF THE SEGMENTATION ALGORITHMS}

The figure 5.8 has shown the all segmentation algorithms which has been implemented to segment the skin lesion for one dermoscopy image such as: Seed region growing based on PSO, Rough fuzzy c-means, Self organizing map (SOM) and Support vector machine (SVM). The figures show that the SRG based on PSO has the best skin lesion segmentation comparing to the

others for this dermoscopy image. More over, the self-organizing map has a better segmentation than the GRFCM and SVM.

1. (SRG +PSO)

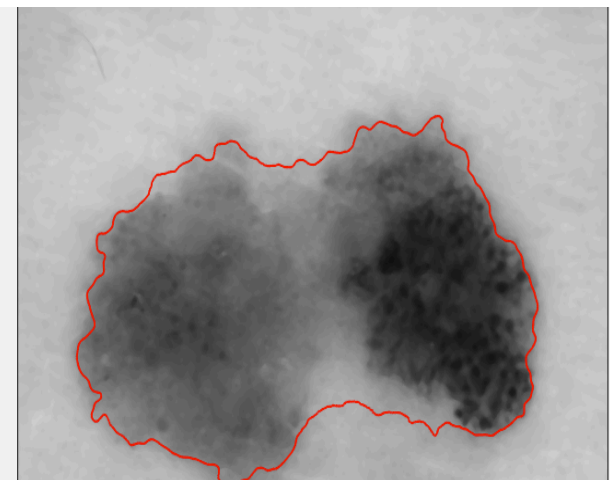

3.(SOM)

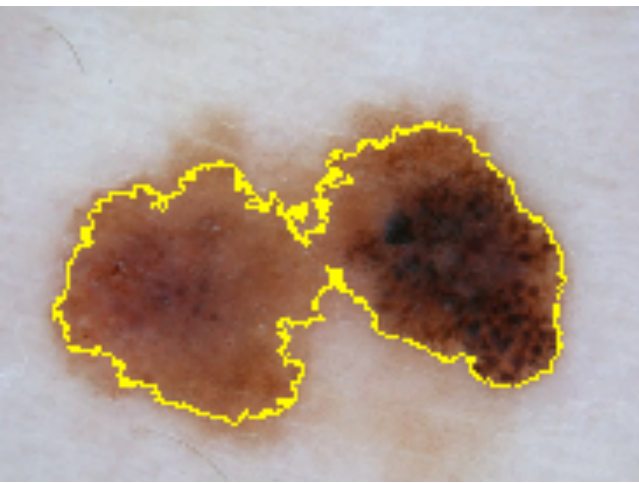

Figure 5.8: The Comparison figure of all segmentation algorithms for one dermoscopy image: SRG based on PSO, Rough fuzzy c-means, SOM \& SVM.
2.(GRFCM algorithm)

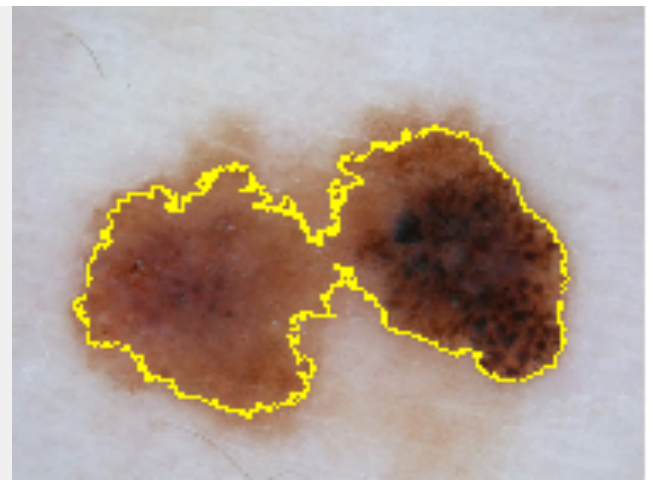

4.(SVM)

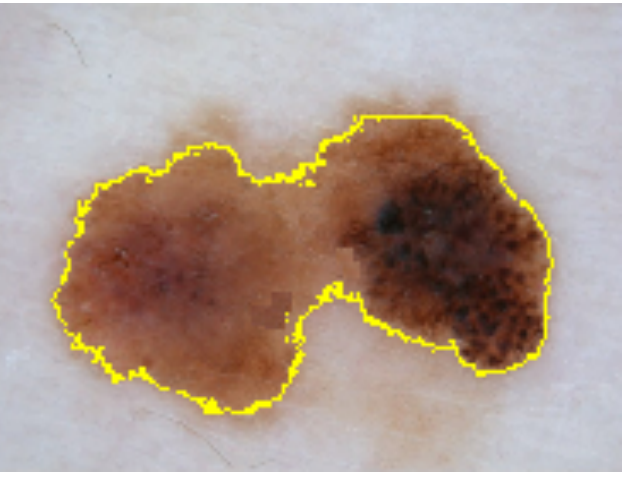


In the final result, we make a comparison table of all the segmentation algorithms for both Derm database (Table 5.1) and ELM and IAD database (Table 5.2). The first Table for Derm database shows that the hybrid algorithms of SRG and PSO can segment the skin lesion with the high accuracy of $90 \%$ and the lesion area segmentation with the accuracy of $88 \%$. After the SRG based on PSO algorithm, SVM, GRFCM and SOM have the highest accuracy of $88 \%, 85 \%$ and $82 \%$ respectively. This order of accuracy is also the same for the lesion area segmentation. It means that the accuracy of the lesion area segmentation for SRG based on PSO, SOM, GRFCM \& SVM is $88 \%, 84 \%, 82 \%$ \& $78 \%$ respectively.

Table 5.1: comparison table of the segmentation algorithms for Derm database
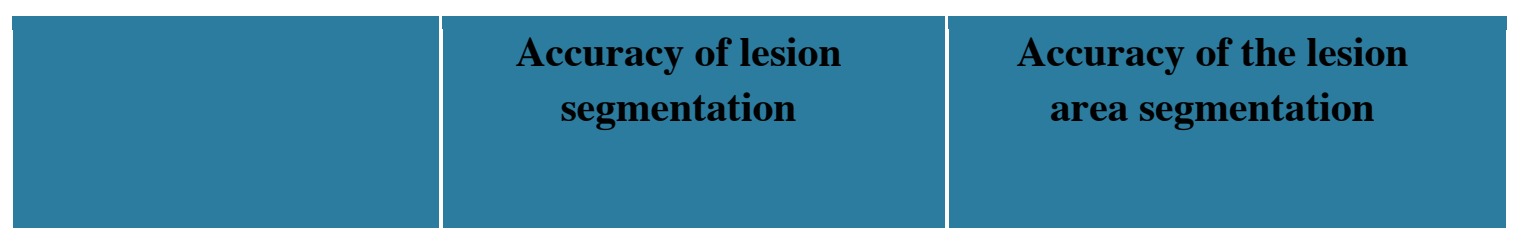

1.SRG +PSO

$90 \%$

$88 \%$

2.SOM

$88 \%$

$84 \%$

3.GRFCM

$85 \%$

$82 \%$

4.SVM

$82 \%$

$78 \%$ 
The second table (Table 5.2) has shown all the segmentation methods for ELM and IAD database and again, The seed region growing based on Particle swarm optimization has the highest accuracy of skin lesion segmentation comparing to the other methods. It also has the sensitivity and specificity of $98 \%$ and $82 \%$ respectively. The second highest performance is belonging to the rough fuzzy c-means with $84 \%$ accuracy and the sensitivity and specificity of $60 \%$ and 90 respectively. SVM has the third highest accuracy of $83 \%$ and the sensitivity of $98 \%$ with the specificity of $80 \%$. SOM has the lowest accuracy of $72 \%$ comparing to the others with the sensitivity of $95 \%$ and specificity of $58 \%$.

Table 5.2: Comparison table of the segmentation algorithms for ELM and IAD database

\begin{tabular}{|l|c|c|c|}
\hline & Accuracy & Sensitivity & Specificity \\
\hline 1.SRG +PSO & $89 \%$ & $98 \%$ & $82 \%$ \\
\hline 2.GRFCM & $84 \%$ & $60 \%$ & $90 \%$ \\
\hline $3 . S V M$ & $83 \%$ & $98 \%$ & $80 \%$ \\
\hline 4.SOM & $72 \%$ & $95 \%$ & $58 \%$ \\
\hline
\end{tabular}




\section{Chapter 6}

\section{CONCLUSIONS \& FUTURE WORKS}

\subsection{SUMMARY AND CONCLUSIONS}

In this project, the goal is to create and implement different new techniques for skin lesion segmentation. In this regard, first, the preprocessing techniques have applied on the images to remove different artifacts and sharpening the image using filtering or the other techniques. The next step, which is the important part of this article, is to present different segmentation algorithms such as: seed region growing based on particle swarm optimization algorithms, rough fuzzy c-means algorithm (GRFCM), support vector machine (SVM) \& self organizing map (SOM). Recently, Hybrid techniques are more accurate \& useful in MRI or the other image segmentation. We used two group datasets of Derm and ELM with IAD images for the skin lesion segmentation. For the evaluation of the segmentation methods, we compare our experimental results with the Ground truth (GT) that is calculated by the radiologist. The results also prove that the SRG based on the PSO, which is a hybrid method, has the highest accuracy comparing with other methods. The proposed approach (GRFCM) avoided the need for manual selection of the suspected region window, seed pixel and threshold value processes, so it has also an acceptable accuracy for the segmentation of the skin lesion.

\subsection{FUTURE WORKS}

For the future works of the project, I can point to some issues. In SRG methods, the results are depending on number of iteration, so its better to create an algorithm, which is not depending of number of iteration. The second one is to work on the deep learning, which is using hierarchical layers of learned abstraction and has recently opened a new dimension on the segmentation of skin lesion. 
The segmentation algorithms still have problem with different shapes, color, texture of the skin and we should create an automated algorithm that the performance of the network is stable and acceptable based on different dermoscopy images, so we can use a combination of a supervised neural network algorithm with different optimization techniques and train the network with a huge dataset of dermoscopy images. More over, we set the parameter of the level set active contour algorithm manually and the result is depending on initial parameters, so its better to create a new method to set the initial parameters automatically. We can also create a userfriendly smart phone application and apply these intelligence segmentation algorithms for diagnosing of melanoma to achieve an accurate result rapidly. For designing a segmentation algorithm, we can create an algorithm to be executable in real-time and also should be optimized for mobile programs as much as possible. 


\section{BIBLIOGRAPHY}

[1] Unsupervised segmentation of skin lesions - Research Gate. Available from: http://www.researchgate.net/publication/33418237_Unsupervised_segmentation_of_ skin_lesions [accessed Nov 16, 2015].

[2] I.S.Akila, V.Sumathi, “ Detection of Melanoma Skin Cancer using Segmentation and Classification Algorithms", National Conference on Information and Communication Technologies (NCICT 2015)

[3] Les Mery, Hannah K. Weir Canadian Cancer Statistics, and Special topic: Skin cancers. Toronto, ON: Canadian Cancer Society; 2014.

[4] Prasan R Bhandari, Varadraj V Pai, "Novel medical strategies combating nonmelanoma skin cancer, Indian Journal of Dermatology", Oct 2014, Volume 59, Issue 6, Page 531-546

[5] DR MCGANN, "MELANOMA- AN IMPROMPTU CONVERSATION" , APRIL 16.2014

[6] Ana Filipa Raimundo, Ricardo Trindade, "classification of skin lesions in dermoscopy images", INSTITUTO SUPERIOR TÉCNICO, Engineering \& medicine, 2012

[7] Maryam Sadeghi, "Towards prevention and early diagnosis of skin cancer: computeraided analysis of dermoscopy images", Simon Fraser University, Summer 2012

[8] M. Emre Celebi, Hitoshi Iyatomi, Gerald Schaefer, "Lesion Border Detection in Dermoscopy Images", Computerized Medical Imaging and Graphics, 33(2): 148153,2010

[9] Nadia Smaoui, Souhir Bessassi, “A developed system for melanoma diagnosis", International Journal of Computer Vision and Signal Processing, 3(1), 10-17(2013)

[10] Fiona M Walter, A Toby Prevost, Joana Vasconcelos, "Using the 7-point checklist as a diagnostic aid for pigmented skin lesions in general practice: a diagnostic validation study", the British journal of general practice, 2013 May; 63(610): e345e353.

[11] Aswin.R.B, J. Abdul Jaleel, Sibi Salim, "Implementation of ANN Classifier using MATLAB for Skin Cancer Detection", International Journal of Computer Science and Mobile Computing, ICMIC13, December- 2013, pg. 87-94 
[12] Ammara Masood and Adel Ali Al-Jumaily, "Computer Aided Diagnostic Support System for Skin Cancer: A Review of Techniques and Algorithms", International Journal of Biomedical Imaging, Volume 2013 (2013), Article ID 323268, 22 pages

[13] Pratt WK. Digital Image Processing: PIKS Inside. Hoboken, NJ: John Wiley \& Sons, 2007.

[14] Delgado D, Butakoff C, Ersboll BK, Stoecker WV. Independent Histogram Pursuit for Segmentation of Skin Lesions. IEEE Trans. on Biomedical Engineering 2008; 55(1): 157-161.

[15] D. Saranya, M. Malini, "A Review of Segmentation Techniques on Melanoma Detection", International Journal of Advanced Research in Computer Science and Software Engineering, Volume 5, Issue 4, 2015

[16] Md.Amran Hossen Bhuiyan, Ibrahim Azad, Md.Kamal Uddin, "Image Processing for Skin Cancer Features Extraction", International Journal of Scientific \& Engineering Research Volume 4, Issue 2, February-2013

[17] Mehemmed emre celebi, "Development of algorithms for dermoscopy image analysis", the university of Texas, Arlington, August 2006

[18] Maryam Sadeghi, "Detection and Analysis of Irregular Streaks in Dermoscopic Images of Skin Lesions", IEEE TRANSACTIONS ON MEDICAL IMAGING, VOL. 32, NO. 5, MAY 2013

[19] Yogendra Kumar Jain, Megha Jain, "Comparison between Different Classification Methods with Application to Skin Cancer", International Journal of Computer Applications (0975 - 8887), Volume 53- No.11, September 2012

[20] I.S.Akila, V.Sumathi, "Detection of Melanoma Skin Cancer using Segmentation and Classification Algorithms", National Conference on Information and Communication Technologies (NCICT 2015)

[21] Kassianos AP, Emery JD, Murchie P, Walter FM" "Smartphone applications for melanoma detection by community, patient and generalist clinician users: a review”, Br J Dermatol. 2015 Jun;172(6):1507-18

[22] Omar Abuzaghleh, Miad Faezipour and Buket D. Barkana, "Skincure: an innovative smart phone-based application to assist in melanoma early detection and prevention, 2015

[23] Q. Abbas, M.E. Celebi, and I.F. Garcia. Hair removal methods: A comparative study for dermoscopy images. Biomedical Signal Processing and Control, 6(4): 395-404, 2011. 
[24] Q. Abbas, I. F. Garcia, and M. Rashid. Unsupervised skin lesions border detection via two- dimensional image analysis. Computer Methods and Programs in Biomedicine, 104(3):e1-e15, 2011.

[25] C.A.Z. Barcelos and V.B. Pires. An automatic based nonlinear diffusion equations scheme for skin lesion segmentation. Applied Mathematics and Computation, 215(1):251-261, 2009.

[26] T.K. Lee, V. Ng, R. Gallagher, A. Coldman, and D. McLean. Dullrazor : A software approach to hair removal from images. Computers in Biology and Medicine, 27(6): 533-543, 1997.

[27] N.H. Nguyena, T.K. Lee, and M.S. Atkinsa. Segmentation of light and dark hair in dermoscopic images: a hybrid approach using a universal kernel. In Proceeding of the SPIE Medical Imaging Conference, volume 7623, pages 76234N-1, 2010.

[28] Wight on, P., Lee, T. K., Lui, H., McLean, D. I., and Atkins, M. S., \Generalizing common tasks in automated skin lesion diagnosis," IEEE Transactions on Information Technology in BioMedicine (2011).

[29] M.E. Celebi, Y.A. Aslandogan, W.V. Stoecker, H. Iyatomi, H. Oka, and X. Chen. Unsuper- vised border detection in dermoscopy images. Skin Research and Technology, 13(4):454-62, 2007.

[30] M.E. Celebi, H. Iyatomi, G. Schaefer, and W.V. Stoecker. Lesion border detection in der- moscopy images. Computerized Medical Imaging and Graphics, 33(2):148 $153,2009$.

[31] M.E. Celebi, H.A. Kingravi, H. Iyatomi, Y.A. Aslandogan, W.V. Stoecker, R.H. Moss, J.M. Malters, J.M. Grichnik, A.A. Marghoob, H.S. Rabinovitz, et al. Border detection in der- moscopy images using statistical region merging. Skin Research and Technology, 14(3):347-353, 2008.

[32] R. Garnavi, M. Aldeen, M.E. Celebi, G. Varigos, and S. Finch. Border detection in der- moscopy images using hybrid thresholding on optimized color channels. Computerized Med- ical Imaging and Graphics, 35(2):105-115, 2011.

[33] H. Iyatomi, H. Oka, M.E. Celebi, M. Hashimoto, M. Hagiwara, M. Tanaka, and K. Ogawa. An improved internet-based melanoma screening system with dermatologist-like tumor area extraction algorithm. Computerized Medical Imaging and Graphics, 32(7):566-579, 2008.

[34] X. Li, B. Aldridge, L. Ballerini, R. Fisher, and J. Rees. Depth data improves skin lesion seg- mentation. Medical Image Computing and Computer-Assisted Intervention-MICCAI 2009, pages 1100-1107, 2009. 
[35] M. Mete, S. Kockara, and K. Aydin. Fast density-based lesion detection in dermoscopy images. Computerized Medical Imaging and Graphics, 35(2):128-136, 2011.

[36] L. Xu, M. Jackowski, A. Goshtasby, D. Roseman, S. Bines, C. Yu, A. Dhawan, and A. Hunt- ley. Segmentation of skin cancer images. Journal of Image and Vision Computing, 17(1):65-74, 1999.

[37] H. Zhou, J.M. Rehg, and M. Chen. Exemplar-based segmentation of pigmented skin lesions from dermoscopy images. In Biomedical Imaging: From Nano to Macro, 2010 IEEE Inter- national Symposium on, pages 225-228. IEEE, 2010.

[38] H. Zhou, G. Schaefer, M.E. Celebi, F. Lin, and T. Liu. Gradient vector flow with mean shift for skin lesion segmentation. Computerized Medical Imaging and Graphics, 35(2):121-127, 2011.

[39] H. Zhou, G. Schaefer, A.H. Sadka, and M.E. Celebi. Anisotropic mean shift based fuzzy c-means segmentation of dermoscopy images. Selected Topics in Signal Processing, IEEE Journal of, 3(1):26-34, 2009.

[40] Gonzalez, R.C., R.E. Woods, and S.L. Eddins, Digital Image Processing Using MATLAB. 2003: Prentice-Hall, Inc.

[41] Lee, W.-L., Chen, Y.-C., Chen, Y.-C., Hsieh, K.-S., Unsupervised segmentation of ultrasonic liver images by multiresolution fractal feature vector. Information Sciences: an International Journal, 2005. 175(3): p. 177-199.

[42] Carvalho, B.M., G.T. Herman, T.Y. Kong, Simultaneous fuzzy segmentation of multiple objects. Discrete Appl. Math., 2005. 151(1-3): p. 55-77.

[43] Liu, H., Xie, C., Chen, Z., Lei, Y.., Segmentation of Ultrasound Image Based on Morphological Operation and Fuzzy Clustering, in Proceedings of the Third IEEE International Workshop on Electronic Design, Test and Applications. 2006, IEEE Computer Society. p. 397-400.

[44] Lung, H.V. and J.-M., Kim, A generalized spatial fuzzy C-means algorithm for medical image segmentation, in Proceedings of the 18th international conference on Fuzzy Systems. 2009, IEEE Press: Jeju Island, Korea. p. 409-414.

[45] Hashemi, S., Kiani, S., Noroozi, N., Moghaddam, M. E., An image contrast enhancement method based on genetic algorithm. Pattern Recogn. Lett., 2010. 31(13): p. 1816-1824.

[46] Lai, C.-C. and C.-Y. Chang, A hierarchical evolutionary algorithm for automatic medical image segmentation. Expert Syst. Appl., 2009. 36(1): p. 248-259. 
[47] Mukhopadhyay, A., U. Maulik, A multiobjective approach to MR brain image segmentation. Appl. Soft Comput., 2011. 11(1): p. 872-880.

[48] M.E. Celebi, H. Iyatomi, G. Schaefer, and W.V. Stoecker. Lesion border detection in der- moscopy images. Computerized Medical Imaging and Graphics, 33(2):148 $153,2009$.

[49] M.E. Celebi, H.A. Kingravi, H. Iyatomi, Y.A. Aslandogan, W.V. Stoecker, R.H. Moss, J.M. Malters, J.M. Grichnik, A.A. Marghoob, H.S. Rabinovitz, et al. Border detection in der- moscopy images using statistical region merging. Skin Research and Technology, 14(3):347-353, 2008.

[50] M.E. Celebi, Y.A. Aslandogan, W.V. Stoecker, H. Iyatomi, H. Oka, and X. Chen. Unsuper- vised border detection in dermoscopy images. Skin Research and Technology, 13(4):454-62, 2007.

[51] H. Iyatomi, H. Oka, M.E. Celebi, M. Hashimoto, M. Hagiwara, M. Tanaka, and K. Ogawa. An improved internet-based melanoma screening system with dermatologist-like tumor area extraction algorithm. Computerized Medical Imaging and Graphics, 32(7):566-579, 2008.

[52] N. Otsu. A threshold selection method from gray-level histograms. IEEE Transactions on Systems, Man and Cybernetic, 9(1):62-66, Jan. 1979.

[53] Celebi, M.E., Sae Hwang; Iyatomi, Hitoshi; Schaefer, G. , "Robust border detection in dermoscopy images using threshold fusion", Image Processing (ICIP), 2010 .

[54] Heydy Castillejos, Volodymyr Ponomaryov, Luis Nino-de-Rivera, and Victor Golikov, "Wavelet Transform Fuzzy Algorithms for Dermoscopic Image Segmentation", Computational and Mathematical Methods in Medicine, Volume 2012, Article ID 578721, 11 pages.

[55] Paul Wighton, Maryam Sadeghi, Tim K. Lee, and M. Stella Atkins, "A Fully Automatic Random Walker Segmentation for Skin Lesions in a Supervised Setting", Med Image Comput Comput Assist Interv. 2009;12(Pt 2):1108-15.

[56] Tingting Liu, Haiyong $\mathrm{Xu}$, Wei Jin, Zhen Liu, Yiming Zhao, and Wenzhe Tian, "Medical Image Segmentation Based on a Hybrid Region-Based Active Contour Model", Computational and Mathematical Methods in Medicine, Volume 2014, Article ID 890725, 10 pages.

[57] M. Emre Celebi, Wenzhao Guo and, "Y. Alp Aslandogan Skin Lesion Segmentation Using Clustering Techniques", American Association for Artificial Intelligence, 2005. 
[58] F. Monteiro, Region-based spatial and temporal image segmentation. PhD thesis, Faculdade de Engenharia da Universidade do Porto, 2007.

[59] Pedro Miguel Martins Ferreira, "Contributions to the segmentation of dermoscopic images", Faculdade de Engenharia da Universidade do Porto, 2012.

[60] I. N. Bankman, Handbook of medical imaging: processing and analysis. Academic Press, 1 ed., 2000.

[61] N. Otsu, "A threshold selection method from gray-level histograms," IEEE Transactions on Systems, Man and Cybernetics, vol. 9, pp. 62-66, January 1979.

[62] Toossi MT, Pourreza HR, Zare H, Sigari MH, Layegh P, Azimi A., "An effective hair removal algorithm for dermoscopy images", Skin Res Technol. 2013 Aug;19(3):230-5. doi: 10.1111/srt.12015. Epub 2013 Apr 7.

[63] Camille Delaporte,"Support Vector machines for lesion Detection in MRI images",MIT student, Virginia Tech,2003.

[64] Harald Galda, Hajime Murao, Hisashi Tamaki," Skin Image Segmentation using a self-organizing map and Genetic algorithms",IEEJ Transactions on Electronics information and systems,01/2003, 123(11).2056-2062.

[65] M.Kanimozhi , CH.Hima Bindu,"Brain MR image Segmentation Using Self organizing Map",International Journal of advanced research in computer \& communication engineering Vol. 2, Issue 10, Oct 2013.

[66] Ali Qusay,Umi Kalthum Ngah, Nor Ashidi Mat Isa,' Breast MRI Tumour Segmentation Using Modified Automatic seeded resion Growing based on particle swarm optimization image clustring",Springer-verlag berlin heidelberg 2011.

[67] Zexuan Ji,Quansen Sun,Yong Xia,'Generalized rough fuzzy c-means algorithm for brain MR image segmentation",computer method and program in biomedicine 108(2012 644-655.

[68] Lahouaoui LALAOUI, Tayeb MOHAMADI, "A comparative study of image region-based segmentation algorithms", (IJACSA) International Journal of Advanced Computer Science and Applications, Vol. 4, No. 6, 2013

[69] Ibrahim, S., et al., Particle Swarm Optimization vs Seed-Based Region Growing: Brain Abnormalities Segmentation. International Journal of Artificial Intelligence, 2011. 7(1): p. 174-188.

[70] Omran, M.G.H., A PSO-based Clustering Algorithm with Application to Unsupervised Image Classification, 2005, University of Pretoria etd. 
[71] Ouadfel, S., M. Batouche, and A. Taleb-Ahmed. A Modified Particle Swarm Optimization Algorithm for Automatic Image Clustering. in International Symposium on Modelling and Implementation of Complex Systems, MISC'2010. 2010.

[72] Wong, M.T., X. He, and W.-C. Yeh, Image Clustering Using Particle Swarm Optimization. 2011.

[73] Adams, R. and L. Bischof, Seeded Region Growing. IEEE Trans. Pattern Anal. Machine Intell, 1994. 16: p. 641-647.

[74] J. A. Nasiri, H. S. Yazdi, and M. Naghibzadeh, "A mouth detection approach based on PSO rule mining on color images," 5th Iranian

[75] C. -C. Hung and W. Li, "Hybridization of particle swarm optimization with the kmeans algorithm for image classification," ISBN 978-1-4244-2760-4/09, 2009.

[76] M. G. H. Omran, A. Salman, and A. P. Engelbrecht, "Dynamic clustering using particle swarm optimization with application in image segmentation," Pattern Analysis Application, 2006, vol. 8, pp. 332-344.

[77] Y. H. Zheng and M. Yan, "The PSO-based adaptive window for people tracking," Proceedings of the 2007 IEEE Symposium on Computational Intelligence in Security and Defense Applications, 2007, pp. 23-29.

[78] Amandeep Kaur, Charanjit Singh, Amandeep Singh Bhandari, "SAR Image Segmentation Based On Hybrid PSOGSA Optimization Algorithm" ,Journal of Engineering Research and Applications, ISSN : 2248-9622, Vol. 4, Issue 9( Version 1), September 2014, pp.05-11

[79] Shahin Ara Begum O. Mema Dev, "A Rough Type-2 Fuzzy Clustering Algorithm for MR Image Segmentation", International Journal of Computer Applications (0975 - 8887) Volume 54- No.4, September 2012

[80] P.Maji,S.K.Pal, "RFCM: a hybrid clustering algorithm using rough and fuzzy sets, Fundam. Inform. 79 (2007) 1-22.

[81] A. Sboner and C. F. Aliferis, "Modeling clinical judgment and implicit guideline compliance in the diagnosis of melanomas using machine learning," in Proceedings of the AMIA Annual Symposium, pp. 664-668, American Medical Informatics Association, 2005.

[82] Jianguo Zhang, Kai-Kuang Ma*, Meng Hwa Er, "Tumor segmentation from magnetic resonance imaging by learning via one-class support vector machine", International Workshop on Advanced Image Technology (IWAIT '04), Jan 2004, Singapore, Singapore. pp.207--211, 2004 
[83] Kohonen, T., Kaski, S., Lagus, K., Salojarvi, J., Honkela, J., Paatero, V., Saarela, A., "Self organization of a massive document collection". IEEE Transactions on Neural Networks 11 (3), (2000), 574-585.

[84] Harald Galda, Hajime Murao, Hisashi Tamaki," Skin Image Segmentation using a self-organizing map and Genetic algorithms",IEEJ Transactions on Electronics information and systems, 01/2003, 123(11).2056-206

[85] Anamika Ahirwar, R.S. Jadon, "Characterization of tumor region using SOM and Neuro Fuzzy techniques in Digital Mammography", International Journal of Computer Science \& Information Technology (IJCSIT), Vol 3, No 1, Feb 2011

[86] M.Kanimozhi , CH.Hima Bindu,"Brain MR image Segmentation Using Self organizing Map",International Journal of advanced research in computer \& communication engineering Vol. 2, Issue 10, Oct 2013

[87] Binder $\mathrm{M}^{1}$, Schwarz M, Winkler A, Steiner A, Kaider A, Wolff K, Pehamberger H., "Epiluminescence microscopy. A useful tool for the diagnosis of pigmented skin lesions for formally trained dermatologists", Arch Dermatol. 1995 Mar;131(3):28691.

[88] A.A. Haseena Thasneem, R. Mehaboobathunnisa , M. Mohammed Sathik and S .Arumugam , "COMPARISON OF DIFFERENT SEGMENTATION ALGORITHMS FOR DERMOSCOPIC IMAGES", ICTACT JOURNAL ON IMAGE AND VIDEO PROCESSING, MAY 2015, VOLUME: 05, ISSUE: 04

[89] Li, C., et al. Level Set Evolution Without Re-initialization: A New Variational Formulation. in IEEE International Conference on Computer Vision and Pattern Recognition (CVPR). 2005. San Diego. 